\title{
Interference Management Schemes for the Shared Relay Concept
}

\author{
Ali Y. Panah, Kien T. Truong, Steven W. Peters, and Robert W. Heath Jr. \\ Department of Electrical and Computer Engineering, The University of Texas at Austin, University Station C0806, \\ Austin, TX 78712-0240, USA \\ Correspondence should be addressed to Ali Y. Panah, ayp@mail.utexas.edu
}

Received 30 June 2010; Accepted 8 September 2010

Academic Editor: Robert Schober

Copyright (c) 2011 Ali Y. Panah et al. This is an open access article distributed under the Creative Commons Attribution License, which permits unrestricted use, distribution, and reproduction in any medium, provided the original work is properly cited.

\begin{abstract}
Sharing a multiantenna relay among several sectors is a simple and cost-effective way to achieving much of the gains of local interference mitigation in cellular networks. Next generation wireless systems, such as ones based on the Third Generation Partnership Projects Long-Term Evolution Advanced, will employ universal frequency reuse to simplify network deployment. This strategy is anticipated to create significant cell-edge interference in the location of the shared relays, thus rendering advanced interference management strategies a necessity. This paper proposes several interference management strategies for the shared relays ranging from simple channel inversion at the relay, to more sophisticated techniques based on channel inversion in combination with partial and full base station coordination in the downlink and uplink. Given that the relay functionality influences total interference, both amplify-and-forward and decode-and-forward type relays are considered throughout. In this context, channel cancelation techniques are investigated for one-way relaying and also the spectrally efficient two-way relaying protocol. Simulations show that strategies based on two-way shared relaying with bidirectional channel inversion at the relay often perform best in terms of total system throughput while one-way techniques are promising when the relay power is low.
\end{abstract}

\section{Introduction}

The IEEE $802.16 \mathrm{j}$ wireless standard was one of the first commercial standards to embrace the use of relay terminals within a cellular network [1]. The use of relay terminals is also provisioned in many upcoming wireless standards such ones emerging from the Third Generation Partnership Program's Long-Term Evolution Advanced (3GPP LTE-A) task group [2-7]. Such deployments are expected to operate under universal frequency reuse patterns so as to maximize area spectral efficiency. Intercell interference, therefore, is omnipresent throughout the network and interference management strategies such as intercell interference coordination [8-12] are of utmost importance in realizing the true gains promised by the standards. While to facilitate interference management, certain means of exchanging information via the X2 interface connecting the base stations have been foreseen in 3GPP LTE-Advanced, practical considerations (such as latency) warrant more research toward interference management at the relay terminals.

Within this context, previously in [7] we evaluated the benefits of several promising relaying strategies for $3 \mathrm{GPP}$
LTE-Advanced including: one-way shared relaying, two-way relaying, and IEEE $802.16 \mathrm{j}$ relaying. Our simulations revealed some key behaviors pertinent to each relaying scheme. The two-way relaying strategy, for instance, exhibited severe interference enhancement in both the uplink and downlink transmissions. This was not surprising since the strategy here was to amplify and forward all received signals at the relays; the amplification process simply did not differentiate between desired signal and interference (or even noise). Even after the subtraction of self-interference (as a benefit to two-way relaying), considerable intersector and intercell interferences aggregated at the receivers. The demodulation processes were subsequently severely degraded, resulting in relevantly low total sum rates. To make matters worse, each sector in each cell contained a two-way relay terminal which individually contributed to such interferences. The "shared relay concept", however, proved to be well suited to handle such interferences, providing adequate sum rate performances comparable even to base station cooperation schemes. Two factors undoubtably attributed to the success of the shared relay concept: (i) interference cancelation: the shared relay did not simply forward signals to the 
destination, it first decoded and demodulated the received signals in the presence of interference, and subsequently forwarded a virtually "interference-free" signal to the destination; a process known as decode and forwarding in relay literature and (ii) minimal infrastructure: unlike the twoway relaying scheme (also the one-way $802.16 \mathrm{j}$ scheme), the shared relay concept, by virtue of its name, was physically shared between several sectors throughout the network. Naturally, less relays were deployed within the network leading not only to possible network cost reduction, but perhaps more importantly the potential to reduce total interference caused by such terminals. As a result, the shared relay concept exhibited a kind resiliency to interference very much desired from a systems design perspective (see, e.g., Figure 8 of [7]). These benefits, however, come at the expense of increased complexity both at the relays, to perform successive interference cancelation, and at the base stations, to perform dirty paper coding. The need for coordination within the shared sectors and issues in synchronization add to these concerns, diminishing the prospects of practical implementation using current hardware capabilities.

In this paper, we expand upon our original shared relay concept to include more intelligent interference management strategies. The main contributions of this paper are as follows. For the one-way shared relay, and in contrast to dirty-paper coding and successive interference cancelation, we reformulate the transmissions to and from the relay to include more practical linear techniques such as zeroforcing precoding and zero-forcing combining (reception). For one-way nonshared (IEEE 802.16j-type) relaying, we include a formulation based on base station coordination via multi-cell cooperative processing, where the coordinated base stations form one virtual antenna array [13-16]. Here, we consider channel inversion (zero-forcing) in the downlink and joint processing to form a multiple-antenna multiple access channels in the uplink. The combination of these strategies improves upon the performance of naive decode and forwarding in our previous work, especially when the receivers are close to the relay terminals. Finally, inspired by observations regarding the original shared relay concept (as briefly touched upon above) the two-way relaying strategy is enhanced in several ways. Firstly, instead of including a relay in each sector of each cell, we resort to a shared two-way relay model. Secondly, we consider interference management, and specifically interference cancelation, at each relay. In this way, the two-way relay will hopefully benefit from the interference cancelation and minimal infrastructure attributes enjoyed by the original shared relay concept.

We also acknowledge, and address, the important fact that the original two-phase two-way protocol has potential power-masking problems, meaning the downlink signals might mask the uplink signals in terms of received power at the relay. This is an artifact of the two-phase protocol where the uplink and downlink signals are received simultaneously at the two-way relay. As a consequence, if the relay makes an effort, for example, to decode the uplink signals, it must do so under extreme interference owing to the downlink transmission. As a remedy, we relax the simultaneous transmission protocol required by the two-way protocol and instead include a three-phase protocol in which the uplink and downlink transmissions are received at different time slots by the relay. While the three-phase protocol takes a hit in terms of multiplexing gain it is still appealing in many ways compared to the two-phase counterpart. A full treatment of this topic is beyond the scope of this paper, we simply note that the three-phase protocol provides the relay with individual processing capabilities of the uplink-downlink signals. As a consequence, the relay has the potential, for example, to distribute its available resources (such as power) differently between the uplink and downlink streams as it broadcasts its common message in the third phase (time slot). The details of this process will become apparent in the two-way relaying section.

The rest of the paper is organized as follows. Section 2 presents the system model while Sections 3 and 4 are devoted to details leading to sum rate expressions for the one and two-way proposed strategies. In Section 5 we present Monte-Carlo simulations assessing the performance of our solutions along with discussion. Finally, Sections 6 and Acknowledgment give summarizing comments and acknowledgments, thus concluding the paper.

This paper uses the following notations. Bold uppercase letters, such as A denote matrices, bold lowercase letters, such as a denote column vectors, and normal letters $a$ denote scalars. The notation $\mathbf{A}^{*}$ denotes the Hermitian transpose of matrix A. The letter $\mathbb{E}$ denotes expectation, $\min \{a, b\}$ denotes the minimum of $a$ and $b,|a|$ is the magnitude of the complex number $a$, and $\|\mathbf{a}\|_{2}^{2}$ is the Euclidean norm of vector $\mathbf{a}$.

\section{System Model}

2.1. General System Model. Consider a network where the cells are labeled by the set $\mathcal{C}=\{1,2, \ldots, C\}$, such that $C=$ $|\mathcal{C}|$ denotes the total number of cells. Each cell contains a single base station (BS) with $N_{t}$ transmit antennas. Moreover, each cell is sectorized and the sectors of the $i$ th cell are labeled by the set $\delta_{i}=\{1,2, \ldots, S\}$, where $S=|\delta|$ is the total number of sectors per cell. For simplicity, we assume equal numbers of BS antennas and sectors in all the cells and that each sector contains a single mobile station (MS). Each BS antenna (corresponding to a sector) transmits one data stream in the downlink (DL) to the MS in its sector and receives a single stream in the uplink from that MS. The $\mathrm{DL} / \mathrm{UL}$ transmissions occur in nonoverlapping time intervals in TDMA fashion, that is, time-sharing.

2.2. Shared Relay Model. At the joint corner of any three adjacent cells there exists a single relay terminal equipped with $N_{r}$ antennas. Such shared relays are labeled by the set $\mathcal{M}=\{1,2, \ldots, M\}$. The purpose of each shared relay is to assist, that is, coordinate, the DL and/or UL transmissions occurring in its assigned adjacent cells.

Specifically, the shared relay assists the transmission in a subset of sectors in the adjacent cells. For example, consider the $m$ th shared relay in coordination with adjacent cells labeled by $\mathcal{A}_{m}=\left\{m_{1}, m_{2}, m_{3}\right\} \subset \mathcal{C}$. Let $\tilde{\vartheta}_{m_{1}} \subseteq s_{m_{1}}, \tilde{\vartheta}_{m_{2}} \subseteq$ $\varsigma_{m_{2}}$ and $\widetilde{\vartheta}_{m_{3}} \subseteq \varsigma_{m_{3}}$ denote subsets of sectors in these cells 


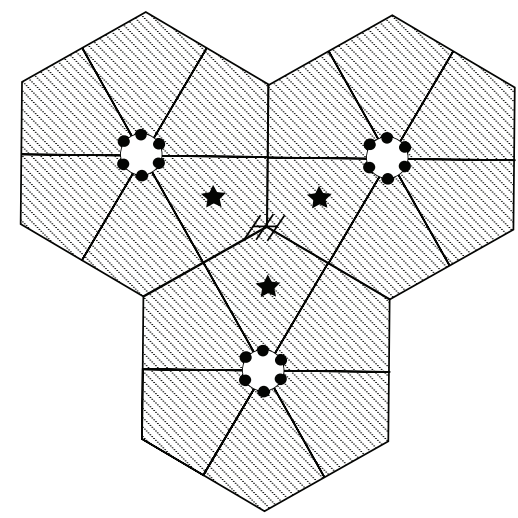

- Base station antennas HH Shared relay stations $\downarrow$ Mobile stations

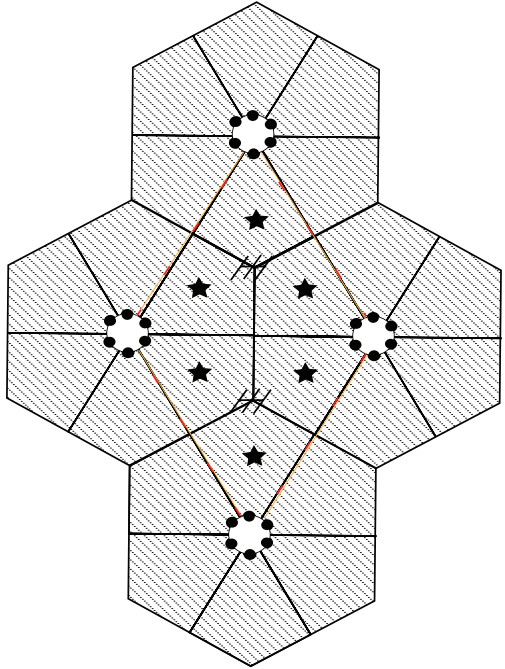

- Base station antennas

H. Shared relay stations

$\downarrow$ Mobile stations

- - - Boundaries of combined sectors served by coordinated BSs

(b)

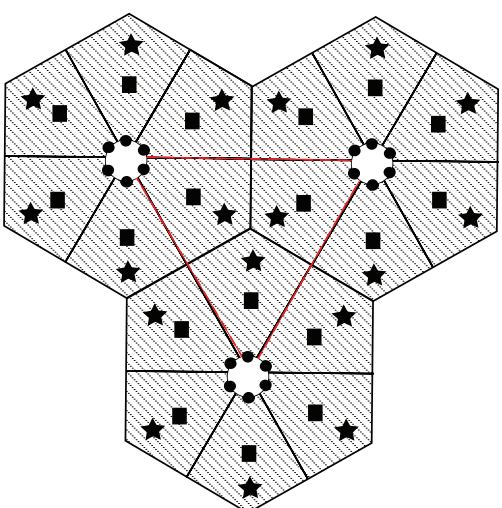

- Base station antennas

- 802.16j-like relay stations

^ Mobile stations

- - - Boundaries of combined sectors served by coordinated BSs

(c)

FIGURE 1: System models for (a) shared relaying (one-way and two-way), (b) shared relaying with BS cooperation (one-way) and (c) nonshared, $802.16 \mathrm{j}$, relaying with BS cooperation.

that are being coordinated. Here, we denote the "sectors of interest" for this shared relay by the set $\widetilde{\Im}_{m}=\tilde{\S}_{m_{1}} \cup \tilde{\S}_{m_{2}} \cup \widetilde{\Im}_{m_{3}}$. For simplicity, we assume henceforth that each shared relay coordinates an equal number of sectors denoted by $N_{c}=$ $\left|\tilde{\S}_{m}\right|, m=1,2, \ldots, M$. Also since we assume that each MS has one antenna, each sector of each BS transmits only a single data stream. Figure 1(a) shows a typical scenario which we consider in our simulations consisting of a 3-cell network $(C=3)$, with each cell sectorized into $S=6$ sectors and three center sectors, that is, $N_{c}=3$, coordinated by a single $(M=1)$ shared relay.

2.3. Nonshared (IEEE 802.16j-type) Relay Model. We describe in this section a scenario where IEEE 802.16jtype relays are used to help the transmission between cooperative base stations and their associate mobile stations. For fair comparison and practicality, we assume localized coordination among the base stations serving the same sectors of interest as in the other architectures. In particular, we assume that there exists a half-duplex decode and forward relay in each sector aiding the data transmission between the base station antenna and one single-antenna mobile station. Moreover, we assume that base station coordination are deployed for intersector interference management (perhaps, intercell interference management if the sectors belong to different cells) for $N_{c}$ adjacent sectors, for example, the three center sectors in Figure 1(c). The $N_{c}$ sectors are of our interest. For notational convenience, the nodes associated with the $k$ th sector of interest are labeled as $\mathrm{BS}_{k}, \mathrm{RS}_{k}$ and $\mathrm{MS}_{k}$ for $k=1, \ldots, N_{c}$. The transmissions in the other sectors are assumed to be uncoordinated and thus cause interference to the signal reception in the $N_{c}$ sectors of interest. Let $N_{i}$ be the number of uncoordinated sectors. We will interchangeably use the terms "802.16j" and "nonshared relay" for modeling this type of relay configuration throughout the paper.

\section{One-Way Relaying Schemes}

In this section, we present two classes of interference management solutions for one-way cellular relaying. In one scheme, which we call one-way shared relaying, the shared relay model as described in Section 2.2 is utilized. The basis for this scheme is the shared relay concept explained in depth in [7], where we evaluated the system employing highcomplexity techniques such as the use of dirty paper coding and joint detection. Here we take a more pragmatic approach to the shared relay concept and formulate the problem using practical transmission-recepetion techniques such as block diagonalization transmission and zero-forcing reception. In this context, we extend the core notion of shared relaying to include more sophisticated transmission schemes that include BS coordination. In yet another scheme, which we simply call one-way nonshared relaying (or 802.16j relaying), we assume that instead of a shared relay, each sector of each cell contains a dedicated relay terminal as explained in Section 2.3; a concept also explained in depth in our previous work [7]. Here, we extend this scheme to include BS coordination as a means of interference management and explain key concepts relating to this configuration. 
3.1. One-Way Shared Relaying with Base Station Coordination. A conventional shared relay serves multiple sectors, communicating with multiple base stations and multiple mobile stations located in different cells. In this manner, a shared relay network operates with less total interference than a conventional tree architecture, where each relay communicates with only one base station, and intercell coordination is very limited. This reduced interference comes with the price of a sophisticated relay with multiple antennas and the ability to communicate using multiuser MIMO techniques. The one-way shared relay transmission protocol was explained in more detail in [7], We begin with a simple nonbasestation-coordination setup similar to the one analyzed in [7], where the transmission protocol was divided into two phases: (i) MIMO multiple access channel (MAC) and (ii) MIMO broadcast channel (BC). We overview each phase separately below and in doing so we introduce various notation used throughout the paper. While our overview is in the context of the DL transmission, the UL treatments follows in a similar fashion and is omitted here.

Multiple Access Channel (MAC). Define $\mathbf{h}_{i j}$ as the length $N_{r}$ channel vector from the BS antenna serving the $j$ th sector of the $i$ th cell to the shared relay and let $s_{i j}$ be the transmitted symbol from this BS antenna. To allow for possible powerloading over the sectors of each BS we let $\mathbb{E}_{s}\left\{s_{i j} s_{i j}^{*}\right\}=P_{i j}^{b}$ and the signals are uncorrelated across the antenna arrays and over the BSs. Consider the $m$ th shared relay, in coordination with cells $\mathcal{A}_{m}$. The sectors of interest, that is, sectors coordinated by the shared relay, are labeled by $\tilde{S}_{m}$. Other sectors belonging to the cells in $\mathcal{A}_{m}$ are termed "intersectors" and are labeled by $\tilde{S}_{m}^{I}$ while cells other than $\mathcal{A}_{m}$ are termed "intercells". The received signal at the shared relay is

$$
\begin{aligned}
\mathbf{y}_{R} & =\sum_{i=1}^{C} \sum_{j=1}^{S} \mathbf{h}_{i j} s_{i j}+\mathbf{n}_{R} \\
& =\sum_{i \in \mathcal{A}_{m}} \sum_{j \in \tilde{S}_{m}} \mathbf{h}_{i j} s_{i j}+\overbrace{\sum_{i \in \mathcal{A}_{m}} \sum_{j \in \tilde{S}_{m}^{I}} \mathbf{h}_{i j} s_{i j}}^{\text {intersector interference }}+\underbrace{\sum_{i \notin \mathcal{A}_{m}} \sum_{j=1}^{S} \mathbf{h}_{i j} s_{i j}}_{\text {intercell interference }}+\mathbf{n}_{R} \\
& =\mathbf{H s}+\boldsymbol{\zeta}_{b}+\mathbf{v}_{b}+\mathbf{n}_{R},
\end{aligned}
$$

where $\mathbf{n}_{R} \sim \mathcal{C} \mathcal{N}\left(\mathbf{0}, N_{0} \mathbf{I}\right)$ is $\mathrm{AWGN}$ at the shared relay. We dropped the relay index $m$ for convenience in the last expression and defined the $N_{r} \times N_{c}$ matrix $\mathbf{H}$ whose columns are constructed from $\mathbf{h}_{i j}$ (for the sectors of interest), and $\mathbf{s}$ as the vector of transmitted symbols from these sectors. The intersector interference (ISI) and intercell (ICI) terms are is collected in $\zeta_{b}$ and $\mathbf{v}_{b}$, respectively.

The relay proceeds to decode the transmitted symbols. With $N_{r} \geq N_{c}$, a zero-forcing (ZF) receiver will use a spatial filter $\mathbf{W}_{\mathrm{DL}, 1}=\mathbf{H}^{\dagger}=\left(\mathbf{H}^{*} \mathbf{H}\right)^{-1} \mathbf{H}^{*}$ to decouple the streams in the sectors of interest and decode the signals from the vector
$\mathbf{W}_{\mathrm{DL}, 1} \mathbf{y}_{R}$. This may be accomplished at an instantaneous sum rate of

$$
R_{1}^{\mathrm{DL}}=\sum_{i=1}^{N_{c}} \log _{2}\left(1+\frac{P_{i}^{b}}{\left[\mathbf{W}_{\mathrm{DL}, 1} \mathbf{q}_{b} \mathbf{q}_{b}^{*} \mathbf{W}_{\mathrm{DL}, 1}^{*}\right]_{i, i}}\right)
$$

where $P_{i}^{b}$ is the power of the $i$ th element of $\mathbf{s}$ and $\mathbf{q}_{b}=$ $\mathbb{E}_{\mathbf{s}}\left\{\boldsymbol{\zeta}_{b} \boldsymbol{\zeta}_{b}^{*}+\mathbf{v}_{b} \mathbf{v}_{b}^{*}\right\}+N_{0} \mathbf{I}_{N_{r}}$ is the interference-plus-noise covariance. The UL is characterized similar to the DL, with the uplink channels (and signals) replacing the downlink ones. For instance the received signal at the relay in the UL is $\mathbf{y}_{R}=\mathbf{G} \mathbf{x}+\zeta_{m}+\mathbf{n}_{R}$, where $\mathbf{G}$ and $\mathbf{x}$ are analogues of $\mathbf{H}$ and $\mathbf{s}$ in the DL. With $\mathbf{W}_{\mathrm{UL}, 1}=\mathbf{G}^{\dagger}=\left(\mathbf{G}^{*} \mathbf{G}^{-1} \mathbf{G}^{*}\right.$ and $\mathbf{q}_{m}=\mathbb{E}_{\mathbf{x}}\left\{\boldsymbol{\zeta}_{m} \zeta_{m}^{*}+\mathbf{v}_{m} \mathbf{v}_{m}^{*}\right\}+N_{0} \mathbf{I}_{N_{r}}$ the UL sum rate in the MAC phase is

$$
R_{1}^{\mathrm{UL}}=\sum_{i=1}^{N_{c}} \log _{2}\left(1+\frac{P^{m}}{\left[\mathbf{W}_{\mathrm{UL}, 1} \mathbf{q}_{m} \mathbf{q}_{m}^{*} \mathbf{W}_{\mathrm{UL}, 1}^{*}\right]_{i, i}}\right),
$$

where $P^{m}$ is the average transmit power of any MS and we collected all transmissions outside the sectors of interest in $\zeta_{m}$.

Broadcast Channel ( $B C)$. Once the relay has decoded the received signals in the sectors of interest it must broadcast the information to the MSs in those sectors. While in [7] we assumed a DPC scheme, here we take a more pragmatic approach and assume a linear precoder at the relay. Specifically, we assume the MSs each have a single antenna and therefore receive a single stream. The precoder at the relay is then designed to cancel, that is, zero force, the channel to the MSs. To this end, define $\mathbf{g}_{i j}$ as the length $N_{r}$ channel vector from the $j$ th MS of the $i$ th cell to the shared relay and assume reciprocal channel so that the channel from the relay to the MSs in the the sectors of interest is $\mathbf{G}^{*}$. Similar to $\mathbf{H}$ (above), the columns of $\mathbf{G}$ are $\mathbf{g}_{i j}$ for sectors indexed by $\tilde{\vartheta}_{m}$. The transmitted signal from the relay is $\mathbf{r}=\mathbf{W}_{\mathrm{DL}, 2} \boldsymbol{\Gamma} \widehat{\mathbf{s}}$, where $\hat{\boldsymbol{s}}$ is the decoded signal (assumed to be correct) with unity energy per element and $\Gamma$ is a diagonal matrix with elements $\gamma_{i}, i=1,2, \ldots, N_{c}$ that controls the power for each element of $\hat{\mathbf{s}}$. Moreover $\boldsymbol{\Gamma}$ is such that the average transmit power of $P^{r}$ is satisfied at the relay. A ZF filter in this case is $\mathbf{W}_{\mathrm{DL}, 2}=\mathbf{G}\left(\mathbf{G}^{*} \mathbf{G}\right)^{-1}$ leading to a sum rate of

$$
R_{2}^{\mathrm{DL}}=\sum_{i=1}^{N_{c}} \log _{2}\left(1+\frac{\gamma_{i}^{2}}{N_{0}}\right) .
$$

The sum rate of the entire communication link from BS to MS in the MAC and BC described above is then

$$
R_{\text {shared }}^{\mathrm{DL}}=\frac{1}{2} \min \left\{R_{1}^{\mathrm{DL}}, R_{2}^{\mathrm{DL}}\right\} .
$$

A similar analysis may be done on the UL to obtain

$$
R_{\text {shared }}^{\mathrm{UL}}=\frac{1}{2} \min \left\{R_{1}^{\mathrm{UL}}, R_{2}^{\mathrm{UL}}\right\},
$$

and the the average sum of the end-to-end rates of both downlink and uplink is $R_{\text {shared }}^{\text {sum }}=R_{\text {shared }}^{\mathrm{DL}}+R_{\text {shared }}^{\mathrm{UL}}$. 
Extension-Base Station Coordination. The shared relay model does not consider base station coordination. Joint reception and transmission of disjoint base stations, however, is becoming a practical option for future generation networks. Thus, shared relays can be envisioned to operate in a network with coordinated base stations, so this section considers such a model for analysis. For this model, we allow multiple base stations to jointly transmit (downlink) or receive (uplink) signals to and from the shared relays and we assume each shared relay still serves $N_{c}$ of the mobile stations (data streams).

In the first hop of the downlink, the model is now a MIMO broadcast channel, rather than a MAC channel in the normal shared relay model. Figure 1(b) shows an embodiment of this scenario where $C=4$ cells, that is, base stations, are connected via a high capacity backhaul link and are able to cooperate in real-time (no delay). Here a total of 6 antennas, that is, $S=6$ sectors, are jointly utilized to transmit 6 streams intended for the indicated $M=2$ shared relays. Each relay will decode $N_{c}=3$ independent streams intended for mobile stations in its sectors of interest. This broadcast channel may readily be realized via block diagonalization. The precoding matrix for shared relay $m$ is in the form of $\mathbf{W}_{\mathrm{BD}}^{(m)}=\widetilde{\mathbf{V}}_{m} \widehat{\mathbf{V}}_{m}$, where $\widetilde{\mathbf{V}}_{m}$ lies in the null space of $\tilde{\mathbf{H}}_{m}=\left[\mathbf{H}_{1}^{*} \cdots \mathbf{H}_{m-1}, \mathbf{H}_{m+1} \cdots \mathbf{H}_{M}^{*}\right]^{*}$, and $\hat{\mathbf{V}}_{m}$ is the matrix with columns of dominant eigenvectors of $\mathbf{H}_{m} \widetilde{\mathbf{V}}_{m}$. In this case each relay will receive $N_{c}$ streams, free of interuser interference. Intersector interference, however, is still present (along with intercell interference) however fewer sectors contribute to such interference since a group of such sectors are now in cooperation. Similar to (1), the received signal at the shared relay is $\mathbf{y}_{R}=\hat{\mathbf{H}} \mathbf{s}+\widetilde{\boldsymbol{\zeta}}_{b}+\widetilde{\mathbf{v}}_{b}+\mathbf{n}_{R}$, where $\widetilde{\boldsymbol{\zeta}}_{b}$ and $\widetilde{\mathbf{v}}_{b}$ are equivalent intersector and intercell interferences. The sum rate at each shared relay is then

$$
R_{1, \text { coop }}^{\mathrm{DL}}=\sum_{i=1}^{N_{c}} \log _{2}\left(1+\frac{P_{i}^{b}}{\left[\tilde{\mathbf{q}}_{b} \tilde{\mathbf{q}}_{b}^{*}\right]_{i, i}}\right),
$$

where $\tilde{\mathbf{q}}_{b}=\mathbb{E}_{s}\left\{\widetilde{\boldsymbol{\zeta}}_{b} \widetilde{\boldsymbol{\zeta}}_{b}^{*}+\tilde{\mathbf{v}}_{b} \widetilde{\mathbf{v}}_{b}^{*}\right\}+N_{0} \mathbf{I}_{N_{r}}$. In the second hop of the downlink, the relays are not able to coordinate their transmissions, so the model resorts to the identical MIMO broadcast channel of the conventional shared relay channel. In other words the relays cannot preform zeroforcing between themselves as was done in the previous phase by the base stations. Thus, the rate in the second hop of the downlink (and, conversely, in the first hop of the uplink) is identical to that of the conventional shared relay channel with zero-forcing precoding given by $(4), R_{2, \text { coop }}^{\mathrm{DL}}=R_{2}^{\mathrm{DL}}$, and the total DL sum rate is

$$
R_{\text {coop }}^{\mathrm{DL}}=\frac{1}{2} \min \left\{R_{1, \text { coop }}^{\mathrm{DL}}, R_{2, \text { coop }}^{\mathrm{DL}}\right\} .
$$

3.2. One-Way Relaying (802.16j-type) with Base Station Coordination. In this section, we compute the sum of the end-to-end achievable rates for both the uplink and the downlink in the model of one-way relaying with base station coordination. This is the (nonshared) 802.16j-type relay model explain in Section 2.3 and in detail in [7]. The coordinated base stations are assumed to share perfectly the data to be transmitted and the knowledge of the channels between base stations and relays via a high-capacity lowdelay wired backhaul link. The information exchange allows for multi-cell cooperative processing, where the coordinated base stations form one virtual antenna array.

We analyze first the downlink transmission. The downlink transmission requires two nonoverlapping stages. In the first stage, the base stations coordinate their transmissions to each relay, forming a multiple-antenna broadcast channel; while in the second stage, the relays decode their intended signals, re-encode and forward to the mobile stations, forming an interference channel. Let $s_{k}$ be the symbol to be transmitted from the $N_{c}$ coordinated base stations antennas to $\mathrm{MS}_{k}$ such that $\mathbb{E}\left\{\left|s_{k}\right|^{2}\right\}=P_{k}^{b}$ and $\mathbb{E}\left\{s_{k} s_{j}^{*}\right\}=0$ for $j \neq k$. We denote $\mathbf{h}_{k}^{*}$, where $\mathbf{h}_{k} \in \mathbb{C}^{N_{c} \times 1}$, as the channel vector from the $K$ coordinated base station antennas to the $k$ th relay. Similarly, let $\boldsymbol{s}_{N_{i}} \in \mathbb{C}^{N_{i} \times 1}$ be the symbol vector to be transmitted from the $N_{i}$ uncoordinated base station antennas to their associate mobile users. We assume that the uncoordinated base station antennas use the same transmit power $P^{b}$, then $\mathbb{E}\left\{\mathbf{s}_{N_{i}} \mathbf{s}_{N_{i}}^{*}\right\}=P^{b} \mathbf{I}$. Also, we denote $\boldsymbol{\theta}_{k}^{*}$, where $\boldsymbol{\theta}_{k} \in \mathbb{C}^{N_{i} \times 1}$, as the channel vector from the $N_{i}$ uncoordinated base station antennas to the $k$ th relay. Moreover, we assume $n_{k} \sim \mathcal{C} \mathcal{N}\left(0, N_{0}\right)$ is the noise vector at the $k$ th relay. For the first stage of the downlink, although achieving the capacity of multiple-antenna broadcast channel, the DPC requires an extensive optimization, leading to significant computational load and overhead. Instead, for simple analysis and practicality, the channel inversion method is employed. We assume $\mathbf{w}_{k} \in \mathbb{C}^{N_{c} \times 1}$ is the beamforming vector corresponding to $s_{k}$. To remove the intersector interference within the cluster of coordinated sectors, we must have $\mathbf{h}_{j}^{*} \mathbf{w}_{k}=0$ for all $j \neq k$, that is, the zero intersector interference constraint. Let us define the combined channel matrix from the $N_{c}$ coordinated base station antennas to the $\left(N_{c}-1\right)$ relays other than the $k$ th relay as

$$
\mathbf{H}_{k}=\left[\begin{array}{llllll}
\mathbf{h}_{1} & \cdots & \mathbf{h}_{k-1} & \mathbf{h}_{k+1} & \cdots & \mathbf{h}_{N_{c}}
\end{array}\right]^{*} .
$$

Under the zero intersector interference constraint and also to maximize the desired signal power, $\mathbf{w}_{k}$ is nothing but the projection of $\mathbf{h}_{k}$ onto the null space of $\mathbf{H}_{k}$. With the set of beamforming vectors, the received signal at the $k$ th relay in the first-hop downlink transmission is written as

$$
r_{k}=\mathbf{h}_{k}^{*} \mathbf{w}_{k} s_{k}+\boldsymbol{\theta}_{k}^{*} \mathbf{s}_{N_{i}}+n_{k} .
$$

The achievable rate of the first-hop downlink transmission from the $N_{c}$ coordinated base station antennas to the $k$ th relay is

$$
R_{1, k}^{\mathrm{DL}}=\log _{2}\left(1+\frac{P_{k}^{b}\left|\mathbf{h}_{k}^{*} \mathbf{w}_{k}\right|^{2}}{P^{b} \boldsymbol{\theta}_{k}^{*} \boldsymbol{\theta}_{k}+N_{0}}\right) .
$$

In the second stage of the downlink transmission, after decoding $s_{k}$, the relay in the $k$ th coordinated sector reencodes it as $x_{k}$ for retransmission to its associate mobile 
station in the same sector. We assume $P_{k}^{r}$ is the transmit power at the relay in the $k$ th coordinated sector. Let $g_{k, j}$ be the channel from the relay in the $j$ th coordinated sector to the mobile user in the $k$ th coordinated sector. Moreover, we denote $\boldsymbol{\beta}_{k}^{*}$, where $\boldsymbol{\beta}_{k} \in \mathbb{C}^{N_{i} \times 1}$, as the channel vector from the relays in the $N_{i}$ uncoordinated sectors to the mobile user in the $k$ th coordinated sectors. We assume that $\mathbf{x}_{N_{i}}$ is the transmitted symbol vectors from the uncoordinated relays. Note that we also have $\mathbb{E}\left\{\mathbf{x}_{N_{i}} \mathbf{x}_{N_{i}}^{*}\right\}=P^{r} \mathbf{I}$, where $P^{r}$ is the transmit power at an uncoordinated relay. Let $v_{k} \sim \mathcal{C} \mathcal{N}\left(0, N_{0}\right)$ be the noise at the mobile user in the $k$ th coordinated sector. The mobile user in the $k$ th coordinated sector receives

$$
y_{k}=g_{k, k} x_{k}+\sum_{j \neq k} g_{k, j} x_{j}+\boldsymbol{\beta}_{k}^{*} \mathbf{x}_{N_{i}}+v_{k} .
$$

The achievable rate of the second-hop downlink transmission in the $k$ th coordinated sector is

$$
R_{2, k}^{\mathrm{DL}}=\log _{2}\left(1+\frac{P_{k}^{r}\left|g_{k, k}\right|^{2}}{\sum_{j \neq k} P_{j}^{r}\left|g_{k, j}\right|^{2}+P^{m} \boldsymbol{\beta}_{k}^{*} \boldsymbol{\beta}_{k}+N_{0}}\right) .
$$

We now analyze the uplink transmission in which the $k$ th mobile station transmits $\tilde{s}_{k}$ to the $k$ th base station. The uplink transmission also requires two stages. In the first stage, the mobile stations transmit signals to the relays, forming an interference channel; and in the second phase, the relays forward the signals to the base stations, which cooperate to perform joint processing to form a multiple-antenna multiple access channel. Let $\tilde{g}_{k, j}$ be the channel from the mobile station in the $j$ th coordinated sector to the relay in the $k$ th coordinated sector and $\phi_{k}^{*}$, where $\phi_{k} \in \mathbb{C}^{N_{i} \times 1}$, be the channel from the mobile users in the $N_{i}$ uncoordinated sectors to the relay in the $k$ th coordinated sector. Similar to the second-hop downlink channel, we obtain the achievable rate of the first-hop uplink channel from the $k$ th mobile station to the $k$ th relay is

$$
R_{1, k}^{\mathrm{UL}}=\log _{2}\left(1+\frac{P_{k}^{m}\left|\tilde{g}_{k, k}\right|^{2}}{\sum_{j \neq k} P_{j}^{m}\left|\tilde{g}_{k, j}\right|^{2}+P^{m} \phi_{k}^{*} \phi_{k}+N_{0}}\right) .
$$

In the second stage of the uplink transmission, we have a multiple-antenna multiple access channel since the base stations can cooperate for joint reception. After decoding $\tilde{s}_{k}$, the $k$ th relay re-encodes it as $\tilde{x}_{k}$ (with $\mathbb{E}\left\{\left|\tilde{x}_{k}\right|^{2}=P_{k}^{r}\right\}$ ) according to the highest rate supported by the transmission from the $k$ th relay to the $N_{c}$ coordinated base station antennas. Let $\tilde{\mathbf{H}}_{k} \in \mathbb{C}^{N_{c} \times N_{c}}$ be the channel matrix from the relays in the $N_{c}$ coordinated sector to the $N_{c}$ coordinated base station antennas. We denote $\boldsymbol{\Psi}_{k} \in \mathbb{C}^{N_{c} \times N_{i}}$ as the channel matrix from the relays in the uncoordinated sectors to the $N_{c}$ coordinated base station antennas and $\tilde{\mathbf{x}}_{N_{i}}$ as the transmitted symbol vector from the relays in the $N_{i}$ uncoordinated sectors. The received signal at the $N_{c}$ coordinated base station antennas is

$$
\tilde{\mathbf{y}}=\tilde{\mathbf{H}}_{k} \widetilde{\mathbf{x}}_{k}+\Psi_{k} \tilde{\mathbf{x}}_{N_{i}}+\mathbf{z}
$$

where $\tilde{\mathbf{x}}_{k}=\left[\tilde{x}_{1} \cdots \tilde{x}_{N_{c}}\right]^{T} \in \mathbb{C}^{N_{c} \times 1}$ and $\mathbf{z}$ is the noise vector at the $N_{c}$ coordinated base station antennas. We assume the zero-forcing receiver $\mathbf{W}=\left(\widetilde{\mathbf{H}}^{*} \tilde{\mathbf{H}}\right)^{-1} \widetilde{\mathbf{H}}$ is applied to $\tilde{\mathbf{y}}$ to decouple the data streams. The achievable data rate in the second-hop of the uplink is given by

$$
R_{2, k}^{\mathrm{UL}}=\log _{2}\left(1+\frac{P_{k}^{r}}{\left[\mathbf{W}\left(\boldsymbol{\Psi}_{k} \mathbf{\Psi}_{k}^{*}+N_{0}\right) \mathbf{W}^{*}\right]_{k, k}}\right) .
$$

We assume $t \in(0,1)$ be the fraction of time used for the firsthop transmission in the downlink and hence $(1-t)$ is that for the second-hop transmission in the downlink. The endto-end achievable rate of the two-hop downlink transmission from the $k$ th base station to the $k$ th mobile station via the $k$ th relay station is $R_{k}^{\mathrm{DL}}=(1 / 2) \min \left\{R_{1, k}^{\mathrm{DL}}, R_{2, k}^{\mathrm{DL}}\right\}$, where for fair comparison with the other approaches in the paper, we assume that equal time sharing for two hops in both directions is used. In other words, we have

$$
R_{\text {nonshared }}^{\mathrm{DL}}=\sum_{k=1}^{N_{c}} \frac{1}{2} \min \left\{R_{1, k}^{\mathrm{DL}}, R_{2, k}^{\mathrm{DL}}\right\} .
$$

This is analogous to (8) for the shared relay model. Similarly, the end-to-end achievable rate in the uplink is $R_{k}^{\mathrm{UL}}=$ $(1 / 2) \min \left\{R_{1, k}^{\mathrm{UL}}, R_{2, k}^{\mathrm{UL}}\right\}$ with

$$
R_{\text {nonshared }}^{\mathrm{UL}}=\sum_{k=1}^{N_{c}} \frac{1}{2} \min \left\{R_{1, k}^{\mathrm{UL}}, R_{2, k}^{\mathrm{UL}}\right\},
$$

and the average sum of the end-to-end rates of both downlink and uplink is $R_{\text {sum }}^{\text {nonshared }}=R_{\text {nonshared }}^{\mathrm{DL}}+R_{\text {nonshared }}^{\mathrm{UL}}$.

\section{Two-Way Relaying Schemes}

In this section, we present three classes of interference management solutions for two-way cellular relaying. Twoway relaying differs from its one-way counterpart mainly in the structure of the UL-DL transmission protocol (see [17-22] for an overview of two-way relaying). Figure 2 highlights this difference, illustrating how the UL and DL transmissions are time-multiplexed (as is assumed in this paper), the one-way relaying scheme requires a total of four time slots while the two-way relaying protocol only requires three. In this regard the two-way protocol is potentially more spectrally efficient than its one-way counterpart. Specifically, one complete UL-DL transmission in the two-way protocol proceeds as follows: (i) the BS transmits a signal to the relay while the MS is silent, that is, the DL, (ii) the MS transmits its signal to the relay while the BS is silent, that is, the UL and (iii) the relay jointly processes the DL and UL signals and proceeds to broadcast a unified signal to the BS and MS. After such, the BS and MS extract their intended signals by first canceling their own transmitted signal which has essentially been "reflected" off the relay. The process of subtracting this so-called self-interferece is crucial to the underlying performance of two-way relaying.

In [7] we proposed a two-way protocol in a cellular setting where we assumed naive signal processing at the 


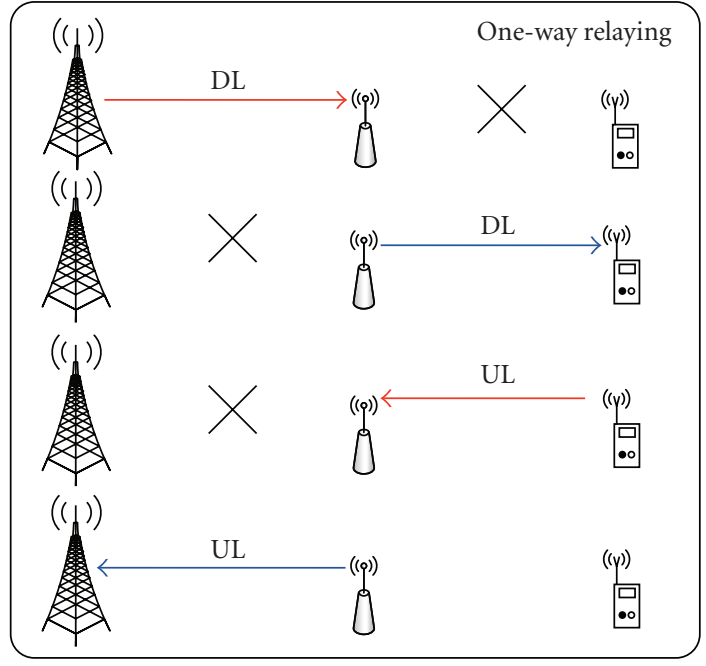

(a)

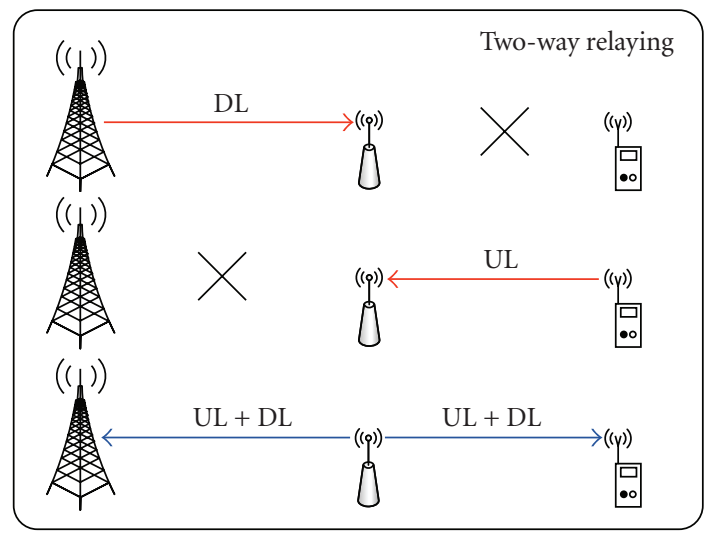

(b)

Figure 2: One-way and two-way transmission protocols.

relay, meaning that no effort was made on dealing with interferences other than removing self-interference inherent to the protocol. As a result the performance of the two-way protocol was severely undermined by intercell and intersector interferences (see, e.g., Figure 8 of [7]). As a remedy, we now propose more sophisticated relay processing techniques tailored for the shared relay model (see Section 2.2). As our simulations show, such efforts may dramatically improve the performance of two-way relaying in interference limited cellular settings.

\subsection{Decode Superimpose Orthogonalize and Forward (DSOF)} Relaying. As a natural extension of the one-way shared relay scheme of Section 3, assume that the shared relay decodes its received signal. In two-way relaying fashion, the following three-phase scheme is proposed.

Phase I-Downlink. the relay receives DL transmission from the sectors of interest labeled by $\tilde{\gamma}_{m}$ while the MSs in these sectors are silent. Denote the received signal in this phase as $y_{R}^{(\mathrm{I})}$ which is exactly (1). In fact this is precisely the MAC phase of the previously discussed one-way shared relay strategy. Again, using a ZF filter to separate the spatial streams from the BS sectors the sum rate of (2) is achievable.

Phase II-Uplink. The roles of the BS and MSs are reversed in the sectors of interest. Denote the received signal in this phase as $\mathbf{y}_{R}^{(\mathrm{II})}=\mathbf{G} \mathbf{x}+\boldsymbol{\zeta}_{m}+\mathbf{n}_{R}$ which is similar to (1) exempt formulated for the UL. The MSs each transmit at a power of $P^{m}$ to the relay thus forming another MAC phase at an achievable rate given by (3).

Phase III-Relay Processing. The relay constructs a single signal to broadcast to both the BS sectors and the MSs (in the sectors of interest). Specifically, after decoding the received signals (assuming the decoding is correct) from phase I and II the relay re-encodes the messages and subsequently pairs the signals by superposition at the signal level. For ease of notation, henceforth consider the three cell network with a central shared relay and sectors of interest as depicted in Figure 1(a). Here, the relay is coordinating one sector in each cell, that is, $\left|\tilde{S}_{m}\right|=1$. Specifically, the relay coordinates with the adjacent sectors of each cell which following the notion of Section 2.2 we assume to be labeled as $\tilde{\boldsymbol{s}}_{m_{1}}=\widetilde{\widetilde{s}}_{m_{2}}=\tilde{\boldsymbol{s}}_{m_{3}}=$ $\{1\}$. Clearly $N_{c}=3$ in this case and the relay constructs the following superposition

$$
\begin{array}{r}
t_{i}=s_{i 1} \sqrt{\frac{1+\gamma}{2}}+x_{i 1} \sqrt{\frac{1-\gamma}{2}}, \quad-1 \leq \gamma \leq 1, \\
i=1,2, \ldots, N_{c}(=3) .
\end{array}
$$

Note how the subscript $i$ denotes a pair of BS-MS in the sector of interest for the $i$ th cell. Next, to spatially separate such BS-MS pairs between the different cells, the relay assigns unique beamforming vectors $\mathbf{w}_{i}$ to each $t_{i}$. The transmitted vector from the relay is $\mathbf{t}_{R}=\sqrt{P^{r}} \sum_{i=1}^{N_{c}} \mathbf{w}_{i} t_{i}=\sqrt{P^{r}} \mathbf{W t}$, where $\mathbf{W} \triangleq\left[\mathbf{w}_{1}, \mathbf{w}_{2}, \ldots, \mathbf{w}_{N_{c}}\right]$ with $\operatorname{tr}\left(\mathbf{W W}^{*}\right)=1, \mathbf{t} \triangleq$ $\left[t_{1}, t_{2}, \ldots, t_{N_{c}}\right]^{T}$, and $P^{r}$ is the total average power from the relay terminal. The signal $\mathbf{t}_{R}$ is broadcasted to the sectors of interest pertaining to the corresponding shared relay. Assuming reciprocity in the channels, the received signal in the sectors of interest in the $i$ th BS is

$$
y_{i}=\mathbf{h}_{i 1}^{*} \mathbf{t}_{R}+n_{i},
$$

where $n_{i} \sim \mathcal{C} \mathcal{N}\left(0, N_{0}\right)$ is AWGN. Similarly, at the $i$ th MS

$$
z_{i}=\mathbf{g}_{i 1}^{*} \mathbf{t}_{R}+v_{i}
$$

where $v_{i} \sim \mathcal{C} \mathcal{N}\left(0, N_{0}\right)$ is AWGN. Viewing these signals in corresponding pairs we define the $2 \times 1$ vector $\mathbf{d}_{i} \triangleq\left[y_{i}, z_{i}\right]^{T}$ so that

$$
\begin{aligned}
\mathbf{d}_{i} & =\left[\begin{array}{ll}
\mathbf{h}_{i 1} & \mathbf{g}_{i 1}
\end{array}\right]^{*} \mathbf{t}_{R}+\left[n_{i}, v_{i}\right]^{T} \\
& =\sqrt{P^{r}} \mathbf{F}_{i} \mathbf{W} \mathbf{t}+\mathbf{n}_{i} \\
& =\sqrt{P^{r}} \mathbf{F}_{i} \mathbf{W}_{i} t_{i}+\sqrt{P^{r}} \sum_{j \neq i} \mathbf{F}_{i} \mathbf{W}_{j} t_{j}+\mathbf{n}_{i j},
\end{aligned}
$$


where $\mathbf{F}_{i} \triangleq\left[\begin{array}{ll}\mathbf{h}_{i 1} & \mathbf{g}_{i 1}\end{array}\right]^{*}$ is a composite BS-MS channel for the $i$ th cell and $\mathbf{n}_{i} \sim \mathcal{C} \mathcal{N}\left(\mathbf{0}, N_{0} \mathbf{I}_{2}\right)$. To enforce spatial separation in (22), that is, cancel the interference from other BS-MS pairs, we set the following constraint on the beamforming vectors $\mathbf{F}_{i} \mathbf{w}_{j}=\mathbf{0}_{2}$, for all $j \neq i$. By defining the $4 \times N_{r}$ matrix $\widetilde{\mathbf{F}}_{i} \triangleq\left[\begin{array}{llllll}\mathbf{F}_{1}^{*} & \cdots & \mathbf{F}_{i-1}^{*} & \mathbf{F}_{i+1}^{*} & \cdots & \mathbf{F}_{3}^{*}\end{array}\right]^{*}$, the beamforming vectors may be obtained from a "block diagonalization" constraint $\widetilde{\mathbf{F}}_{i} \mathbf{w}_{i}=\mathbf{0}_{4}, i=1,2,3$. Denote the SVD of $\widetilde{\mathbf{F}}_{i}$ as $\mathbf{U}_{i}\left[\begin{array}{ll}\boldsymbol{\Sigma}_{i} & \mathbf{0}_{4 \times M}\end{array}\right] \mathbf{V}_{i}^{*}$, where $\mathbf{V}_{i}=\left[\begin{array}{ll}\mathbf{V}_{i}^{(i)} & \mathbf{V}_{i}^{(0)}\end{array}\right]$ and $\mathbf{U}_{i}$ are unitary matrices, $\boldsymbol{\Sigma}_{i}$ is a $4 \times 4$ diagonal matrix with nonzero elements and the columns of $\mathbf{V}_{i}^{(1)}$ are the corresponding right singular vectors. The $N_{r} \times\left(N_{r}-4\right)$ matrix $\mathbf{V}_{i}^{(0)}$ represents the nullspace of $\widetilde{\mathbf{F}}_{i}$ which for $N_{r}=5$ consist of a single column vector that may be chosen for the beamforming vector $\mathbf{w}_{i}$ (with normalization by $\sqrt{3}$ to preserve the power constraint since $\left\|\mathbf{V}_{i}^{(0)}\right\|_{2}^{2}=1$ ). With this solution (22) reduces to $\mathbf{d}_{i}=$ $\mathbf{F}_{i} \mathbf{w}_{i} t_{i}+\mathbf{n}_{i}$. The self-interference is manifested in the received signals by substituting for the superposition from (19) into (20) and (21). For example, at the BSs we have

$$
\begin{aligned}
y_{i} & =\sqrt{P^{r}} \mathbf{h}_{i 1}^{*} \mathbf{w}_{i} t_{i}+n_{i} \\
& =\sqrt{\frac{P^{r}}{2}} \mathbf{h}_{i 1}^{*} \mathbf{w}_{i}\left(\sqrt{1+\gamma} s_{i 1}+\sqrt{1-\gamma} x_{i 1}\right)+n_{i} \\
& =\underbrace{\sqrt{\frac{P^{r}(1+\gamma)}{2}} \mathbf{h}_{i 1}^{*} \mathbf{w}_{i} s_{i 1}}_{\text {self-interference }}+\underbrace{\sqrt{\frac{P^{r}(1-\gamma)}{2}} \mathbf{h}_{i 1}^{*} \mathbf{w}_{i} x_{i 1}}_{\text {desired }}+n_{i},
\end{aligned}
$$

such that the desired signal from the MS may be detected from $\tilde{y}_{i}=y_{i}-\sqrt{P^{r}(1+\gamma) / 2} \mathbf{h}_{i 1}^{*} \mathbf{w}_{i} s_{i 1}$. The uplink sum rate in this third phase is then

$$
R_{3}^{\mathrm{UL}}=\sum_{i=1}^{N_{c}} \log _{2}\left(1+\frac{P^{r}(1-\gamma)}{2 N_{0}} \mathbf{h}_{i 1}^{*} \mathbf{w}_{i} \mathbf{w}_{i}^{*} \mathbf{h}_{i 1}\right) .
$$

Similarly at the MS, detection of the signal from the BS (via the relay) may be obtained from $\tilde{z}_{i}=z_{i}-$ $\sqrt{P^{r}(1-\gamma) / 2} \mathbf{g}_{i 1}^{*} \mathbf{w}_{i} x_{i 1}$, and the downlink sum rate is

$$
R_{3}^{\mathrm{DL}}=\sum_{i=1}^{N_{c}} \log _{2}\left(1+\frac{P^{r}(1+\gamma)}{2 N_{0}} \mathbf{g}_{i 1}^{*} \mathbf{w}_{i} \mathbf{w}_{i}^{*} \mathbf{g}_{i 1}\right)
$$

Combining (2), (3), (25) and (24), the uplink and downlink sum rates are given by

$$
\begin{aligned}
& R_{\mathrm{DSOF}}^{\mathrm{DL}}=\frac{1}{3} \min \left\{R_{1}^{\mathrm{DL}}, R_{3}^{\mathrm{DL}}\right\}, \\
& R_{\mathrm{DSOF}}^{\mathrm{UL}}=\frac{1}{3} \min \left\{R_{2}^{\mathrm{UL}}, R_{3}^{\mathrm{UL}}\right\} .
\end{aligned}
$$

4.2. Amplify Superimpose and Forward (ASF) Relaying. A less sophisticated relay may choose not to decode the symbols in phase I and II but instead form a scaled superposition $\mathbf{t}_{R}=$ $\mu_{d} \mathbf{y}_{R}^{(\mathrm{I})}+\mu_{u} \mathbf{y}_{R}^{(\mathrm{II})}$ to broadcast in the third phase, where $\mu_{u}, \mu_{d}>$ 0 are a scalers chosen such that the average power constraint
$\mathbb{E}\left\{\operatorname{tr}\left(\mathbf{t}_{R} \mathbf{t}_{R}^{*}\right)\right\}=P^{r}$ is not violated at the relay. To allow for a fair comparison with previous relay strategies while satisfying the power constraint for this scheme we set

$$
\begin{gathered}
\frac{\mu_{d}^{2}\left\|\mathbf{y}_{R}^{(\mathrm{I})}\right\|_{2}^{2}}{\mu_{d}^{2}\left\|\mathbf{y}_{R}^{(\mathrm{II})}\right\|_{2}^{2}}=\frac{1+\gamma}{1-\gamma}, \\
P^{r}=\mu_{d}^{2}\left\|\mathbf{y}_{R}^{(\mathrm{I})}\right\|_{2}^{2}+\mu_{u}^{2}\left\|\mathbf{y}_{R}^{(\mathrm{II})}\right\|_{2}^{2},
\end{gathered}
$$

where $-1 \leq \gamma \leq 1$. Combining these conditions we have

$$
\mu_{d}=\sqrt{\left(\frac{1+\gamma}{2}\right) \frac{P^{r}}{\left\|\mathbf{y}_{R}^{(\mathrm{I})}\right\|_{2}^{2}}}, \quad \mu_{u}=\sqrt{\left(\frac{1-\gamma}{2}\right) \frac{P^{r}}{\left\|\mathbf{y}_{R}^{(\mathrm{II})}\right\|_{2}^{2}}},
$$

which by substitution from $\mathbf{y}_{R}^{(\mathrm{I})}$ and $\mathbf{y}_{R}^{(\mathrm{II})}$ simplifies to

$$
\begin{aligned}
& \mu_{d}=\sqrt{\left(\frac{1+\gamma}{2}\right) \frac{P^{r}}{P_{b}\|\mathbf{H}\|_{F}^{2}+\operatorname{tr}\left(\zeta_{b}^{*} \zeta_{b}\right)+N_{r} N_{0}}}, \\
& \mu_{u}=\sqrt{\left(\frac{1-\gamma}{2}\right) \frac{P^{r}}{P_{m}\|\mathbf{G}\|_{F}^{2}+\operatorname{tr}\left(\zeta_{m}^{*} \zeta_{m}\right)+N_{r} N_{0}}} .
\end{aligned}
$$

Using $\mathbf{y}_{R}^{(\mathrm{I})}$ and $\mathbf{y}_{R}^{(\mathrm{II})}$ we have

$$
\mathbf{t}_{R}=\mu_{d} \sum_{i=1}^{N_{c}} \sum_{j=1}^{S} \mathbf{h}_{i j} s_{i j}+\mu_{d} \mathbf{n}_{R}^{(\mathrm{I})}+\mu_{u} \sum_{i=1}^{N_{c}} \sum_{j=1}^{S} \mathbf{g}_{i j} x_{i j}+\mu_{u} \mathbf{n}_{R}^{(\mathrm{II})} .
$$

Assuming reciprocity in the channels, the received signal in first sector of the ith BS after phase III is

$$
\begin{aligned}
y_{i}= & \mathbf{h}_{i 1}^{*} \mathbf{t}_{R}+n_{i} \\
= & \mu_{d} \mathbf{h}_{i 1}^{*} \sum_{i=1}^{N_{c}} \sum_{j=1}^{S} \mathbf{h}_{i j} s_{i j}+\mu_{u} \mathbf{h}_{i 1}^{*} \sum_{i=1}^{N_{c}} \sum_{j=1}^{S} \mathbf{g}_{i j} x_{i j}+\tilde{n}_{i} \\
= & \underbrace{\mu_{d} \sum_{j=1}^{6} \mathbf{h}_{i 1}^{*} \mathbf{h}_{i j} s_{i j}}_{\text {self-interference }}+\underbrace{\mu_{u} \mathbf{h}_{i 1}^{*} \mathbf{g}_{i 1} x_{i 1}}_{\text {desired signal }}+\underbrace{\mu_{u} \mathbf{h}_{i 1}^{*} \sum_{j=2}^{S} \mathbf{g}_{i j} x_{i j}}_{\text {a priori decoded }} \\
& +\zeta_{b}^{\prime}+\zeta_{m}^{\prime}+\tilde{n}_{i},
\end{aligned}
$$

where $n_{i} \sim \mathcal{C} \mathcal{N}\left(0, N_{0}\right)$ is AWGN, $\tilde{n}_{i} \sim \mathcal{C} \mathcal{N}\left(0, N_{0}(1+\right.$ $\left.\left.\left(\mu_{d}^{2}+\mu_{u}^{2}\right)\left\|\mathbf{h}_{i 1}\right\|_{2}^{2}\right)\right)$. We highlighted a portion as "a priori decoded" meaning it can be subtracted from $y_{i}$ without error. This is reasonable since this term relates to intraMS transmissions within the cell that are not utilizing the shared relay and hence may be decoded in (for example) phase II of the three-phase protocol. Also, $\zeta_{b}^{\prime}$ and $\zeta_{m}^{\prime}$ are intercell BS and MS interferences, respectively, where $\zeta_{b}^{\prime}=$ $\mu_{d} \mathbf{h}_{i 1}^{*} \sum_{k \neq i} \sum_{j=1}^{6} \mathbf{h}_{k j} s_{k j}$ and $\zeta_{m}^{\prime}=\mu_{u} \mathbf{h}_{i 1}^{*} \sum_{k \neq i}^{3} \sum_{j=1}^{S} \mathbf{g}_{k j} x_{k j}$. 
The transmission rate from the $i$ th MS may be obtained after removing the self-interference and the uplink sum rate is obtained as

$$
\begin{aligned}
& R_{\mathrm{ASF}}^{\mathrm{UL}} \\
& =\frac{1}{3} \sum_{i=1}^{N_{c}} \log _{2}\left(1+\frac{P^{m} \mu_{u}^{2}\left|\mathbf{h}_{i 1}^{*} \mathbf{g}_{i 1}\right|^{2}}{N_{0}+N_{0}\left(\mu_{d}^{2}+\mu_{u}^{2}\right)\left\|\mathbf{h}_{i 1}\right\|_{2}^{2}+\left|\zeta_{b}^{\prime}\right|^{2}+\left|\zeta_{m}^{\prime}\right|^{2}}\right) .
\end{aligned}
$$

Similarly, in the downlink we have

$$
\begin{aligned}
z_{i} & =\mathbf{g}_{i 1}^{*} \mathbf{t}_{R}+v_{i} \\
& =\underbrace{\mu_{u} \mathbf{g}_{i 1}^{*} \mathbf{g}_{i 1} x_{i 1}}_{\text {self-interference }}+\underbrace{\mu_{d} \mathbf{g}_{i 1}^{*} \mathbf{h}_{i 1} s_{i 1}}_{\text {desired signal }}+\zeta_{b}^{\prime \prime}+\zeta_{m}^{\prime \prime}+\tilde{n}_{i},
\end{aligned}
$$

where $\tilde{n}_{i} \sim \mathcal{C} \mathcal{N}\left(0, N_{0}\left(1+\left(\mu_{d}^{2}+\mu_{u}^{2}\right)\left\|\mathbf{g}_{i 1}\right\|_{2}^{2}\right)\right)$ and $\zeta_{b}^{\prime \prime}=$ $\mu_{d} \mathbf{g}_{i 1}^{*} \sum_{i=1}^{N_{c}} \sum_{j=1}^{S} \mathbf{h}_{i j} s_{i j}-\mu_{d} \mathbf{g}_{i 1}^{*} \mathbf{h}_{i 1} s_{i 1}$ and $\zeta_{m}^{\prime \prime}=\mu_{u} \mathbf{g}_{i 1}^{*} \sum_{i=1}^{N_{c}} \sum_{j=1}^{S}$ $\times \mathbf{g}_{i j} x_{i j}-\mu_{u} \mathbf{g}_{i 1}^{*} \mathbf{g}_{i 1} x_{i 1}$

$$
\begin{aligned}
& R_{\mathrm{ASF}}^{\mathrm{DL}} \\
& =\frac{1}{3} \sum_{i=1}^{N_{c}} \log _{2}\left(1+\frac{P_{b} \mu_{d}^{2}\left|\mathbf{g}_{i 1}^{*} \mathbf{h}_{i 1}\right|^{2}}{N_{0}+N_{0}\left(\mu_{d}^{2}+\mu_{u}^{2}\right)|| \mathbf{g}_{i 1}||_{2}^{2}+\left|\zeta_{b}^{\prime \prime}\right|^{2}+\left|\zeta_{m}^{\prime \prime}\right|^{2}}\right) .
\end{aligned}
$$

In summary, the ASF strategy reduces potential interference via the subtraction of "a priori decoded" signals. While this process is performed at the BSs, the relay terminal opts for a rather naive approach to signal reception by simply adding the UL/DL signals. The next strategy proposes more aggressive interference management at the relay, while maintaining the amplify and forward nature of the relay.

4.3. Amplify Superimpose Orthogonalize and Forward (ASOF) Relaying. The interference from other sectors of interest in (32) may be eliminated by using a pair of zero-forcing precoders, $\mathbf{A}_{d}$ and $\mathbf{A}_{u}$, at the relay such that the composite channels to the relay are orthogonalized. We call this scheme the amplify superimpose orthogonalize and forward (ASOF) scheme. The relay first linearly precodes the uplink and downlink streams to construct $\mathbf{t}=\mathbf{A}_{d} \mathbf{y}_{R}^{(\mathrm{I})}+\mathbf{A}_{u} \mathbf{y}_{R}^{(\mathrm{II})}$ where $\mathbf{A}_{d}$ and $\mathbf{A}_{d}$ are full-rank $N_{r} \times N_{r}$ matrices that process the downlink and uplink streams, respectively. Substituting for $\mathbf{y}_{R}^{(\mathrm{I})}$ and $\mathbf{y}_{R}^{(\mathrm{II})}$ we have

$$
\begin{aligned}
\mathbf{t} & =\mathbf{A}_{d} \mathbf{H} \mathbf{s}+\mathbf{A}_{d} \boldsymbol{\zeta}_{b}+\mathbf{A}_{d} \mathbf{n}_{R}^{(\mathrm{I})}+\mathbf{A}_{u} \mathbf{G} \mathbf{x}+\mathbf{A}_{u} \boldsymbol{\zeta}_{m}+\mathbf{A}_{u} \mathbf{n}_{R}^{(\mathrm{II})} \\
& =\mathbf{A}_{d} \mathbf{H} \mathbf{s}+\mathbf{A}_{u} \mathbf{G} \mathbf{x}+\tilde{\mathbf{n}}_{R}
\end{aligned}
$$

where $\tilde{\mathbf{n}}_{R}=\mathbf{A}_{d} \mathbf{n}_{R}^{(\mathrm{I})}+\mathbf{A}_{u} \mathbf{n}_{R}^{(\mathrm{II})}+\mathbf{A}_{u} \boldsymbol{\zeta}_{m}+\mathbf{A}_{d} \boldsymbol{\zeta}_{b}$. Setting $\mathbf{A}_{d}=$ $a_{d} \mathbf{H}^{\dagger}=\left(\mathbf{H}^{*} \mathbf{H}\right)^{-1} \mathbf{H}^{*}$ and $\mathbf{A}_{u}=a_{u} \mathbf{G}^{\dagger}=a_{u}\left(\mathbf{G}^{*} \mathbf{G}\right)^{-1} \mathbf{G}^{*}$, the channels to the relay in phase I and II are equalized such that $\mathbf{t}=a_{d} \mathbf{s}+a_{u} \mathbf{x}+\tilde{\mathbf{n}}_{R}$.

Next, a common transmit precoder $\mathbf{W}$ is used to spatially separate the BS-MS pairs such that the transmitted vector from the relay is $\mathbf{t}_{R} \triangleq \mathbf{W t}$, where $\mathbf{W} \triangleq\left[\mathbf{w}_{1}, \mathbf{w}_{2}, \mathbf{w}_{3}\right]$ with $\operatorname{tr}\left(\mathbf{W W}^{*}\right)=1$. The design of $\mathbf{W}$ is identical to the blockdiagonalization explained before. At the BSs we have

$$
\begin{aligned}
y_{i} & =\mathbf{h}_{i 1}^{*} \mathbf{w}_{i} t_{i}+\mathbf{h}_{i 1}^{*} \mathbf{W} \tilde{\mathbf{n}}_{R}+n_{i} \\
& =\mathbf{h}_{i 1}^{*} \mathbf{w}_{i}\left(a_{d} s_{i 1}+a_{u} x_{i 1}\right)+\tilde{n}_{i} \\
& =\underbrace{a_{d} \mathbf{h}_{i 1}^{*} \mathbf{w}_{i} s_{i 1}}_{\text {self-interference }}+\underbrace{a_{u} \mathbf{h}_{i 1}^{*} \mathbf{w}_{i} x_{i 1}}_{\text {desired }}+\tilde{n}_{i} .
\end{aligned}
$$

The uplink sum rate is then

$$
R_{\mathrm{ASOF}}^{\mathrm{UL}}=\frac{1}{3} \sum_{i=1}^{N_{c}} \log _{2}\left(1+\frac{P^{m} a_{u}^{2} \mathbf{h}_{i 1}^{*} \mathbf{w}_{i} \mathbf{w}_{i}^{*} \mathbf{h}_{i 1}}{N_{0}+\mathbf{h}_{i 1}^{*} \mathbf{W}(\boldsymbol{Q}) \mathbf{W}^{*} \mathbf{h}_{i 1}}\right),
$$

where $\boldsymbol{Q}$ denotes $\mathbf{A}_{d} \mathbf{A}_{d}^{*} N_{0}+\mathbf{A}_{u} \mathbf{A}_{u}^{*} N_{0}+\mathbf{A}_{d} \boldsymbol{\zeta}_{b} \boldsymbol{\zeta}_{b}^{*} \mathbf{A}_{d}^{*}+$ $\mathbf{A}_{u} \zeta_{m} \zeta_{m}^{*} \mathbf{A}_{u}^{*}$, Similarly the downlink sum rate is

$$
R_{\mathrm{ASOF}}^{\mathrm{DL}}=\frac{1}{3} \sum_{i=1}^{N_{c}} \log _{2}\left(1+\frac{P^{b} a_{d}^{2} \mathbf{g}_{i 1}^{*} \mathbf{w}_{i} \mathbf{w}_{i}^{*} \mathbf{g}_{i 1}}{N_{0}+\mathbf{g}_{i 1}^{*} \mathbf{W}(\mathcal{Q}) \mathbf{W}^{*} \mathbf{g}_{i 1}}\right),
$$

where $Q$ denotes $\mathbf{A}_{d} \mathbf{A}_{d}^{*} N_{0}+\mathbf{A}_{u} \mathbf{A}_{u}^{*} N_{0}+\mathbf{A}_{d} \boldsymbol{\zeta}_{b} \boldsymbol{\zeta}_{b}^{*} \mathbf{A}_{d}^{*}+$ $\mathbf{A}_{u} \boldsymbol{\zeta}_{m} \boldsymbol{\zeta}_{m}^{*} \mathbf{A}_{u}^{*}$. Finally, Section 6 gives summarizing comments concluding the paper. Noting that $P^{r}=\mathbb{E}\left\{\left\|\mathbf{t}_{R}\right\|_{2}^{2}\right\}=\mathbb{E}\left\{\|\mathbf{t}\|_{2}^{2}\right\}$ the scalers $a_{d}$ and $a_{u}$ are determined similar to (30) as

$$
\begin{gathered}
\frac{a_{d}^{2}\left\|\mathbf{H}^{\dagger} \mathbf{y}_{R}^{(\mathrm{I})}\right\|_{2}^{2}}{a_{u}^{2}\left\|\mathbf{G}^{\dagger} \mathbf{y}_{R}^{(\mathrm{II})}\right\|_{2}^{2}}=\frac{1+\gamma}{1-\gamma}, \\
P^{r}=a_{d}^{2}\left\|\mathbf{H}^{\dagger} \mathbf{y}_{R}^{(\mathrm{I})}\right\|_{2}^{2}+a_{u}^{2}\left\|\mathbf{G}^{\dagger} \mathbf{y}_{R}^{(\mathrm{II})}\right\|_{2}^{2},
\end{gathered}
$$

where $-1 \leq \gamma \leq 1$. Combining these conditions we have

$a_{d}=\sqrt{\left(\frac{1+\gamma}{2}\right) \frac{P^{r}}{\left\|\mathbf{H}^{\dagger} \mathbf{y}_{R}^{(\mathrm{I})}\right\|_{2}^{2}}}, \quad \mu_{u}=\sqrt{\left(\frac{1-\gamma}{2}\right) \frac{P^{r}}{\left\|\mathbf{G}^{\dagger} \mathbf{y}_{R}^{(\mathrm{II})}\right\|_{2}^{2}}}$

which by substitution for $\mathbf{y}_{R}^{(\mathrm{I})}$ and $\mathbf{y}_{R}^{(\mathrm{II})}$ simplifies to

$$
\begin{aligned}
& a_{d}=\sqrt{\left(\frac{1+\gamma}{2}\right) \frac{P^{r}}{N_{c} P^{b}+\operatorname{tr}\left(\mathbf{H}\left(\mathbf{H}^{*} \mathbf{H}\right)^{-2} \mathbf{H}^{*}\left(\boldsymbol{\zeta}_{b} \boldsymbol{\zeta}_{b}^{*}+N_{0} \mathbf{I}_{M}\right)\right)}}, \\
& a_{u}=\sqrt{\left(\frac{1-\gamma}{2}\right) \frac{P^{r}}{N_{c} P^{m}+\operatorname{tr}\left(\mathbf{G}\left(\mathbf{G}^{*} \mathbf{G}\right)^{-2} \mathbf{G}^{*}\left(\zeta_{m} \boldsymbol{\zeta}_{m}^{*}+N_{0} \mathbf{I}_{M}\right)\right)}} .
\end{aligned}
$$

\section{Numerical Results}

The above schemes were simulated under system conditions similar to [7], and without a direct link. Starting with the basic 3-cell cellular topology of the shared relay concept in Figure 1(a), BS coordination is added as in Figure 1(b) to form the basis of the first proposed scheme of Section 3. Figure 1(c) shows the system topology used to simulate the 
TABLE 1: Parameters for multi-cell simulation.

\begin{tabular}{lc}
\hline BS transmit power & $47 \mathrm{dBm}$ \\
MS transmit power & $24 \mathrm{dBm}$ \\
RS transmit power & $5 \sim 37 \mathrm{dBm}$ \\
Noise power (AWGN) & $-109 \mathrm{dBm}$ \\
Sectors per BS & 6 \\
Frequency reuse factor & 1 \\
BS-RS model (NLOS) & IEEE $802.16 \mathrm{j}(\mathrm{H})$ \\
RS-MS model (NLOS) & IEEE $802.16 \mathrm{j}(\mathrm{E})$ \\
Cell radius & $876 \mathrm{~m}$ \\
Building separation & $30 \mathrm{~m}$ \\
MS height & $1 \mathrm{~m}$ \\
RS height & $15 \mathrm{~m}$ \\
BS height & $30 \mathrm{~m}$ \\
Carrier frequency & $2 \mathrm{GHz}$ \\
City environment & $\mathrm{Urban}$ \\
\hline
\end{tabular}

nonshared scheme. Although Figure 1(a) was introduced for one-way relaying it also serves as the system model for the two-way schemes of Section 4, where instead the relay is operating as a bidirectional terminal. Regardless of the scheme, we are interested in the uplink and downlink sum rate performances of the schemes in the sectors of interest which are sectors in which all three base stations share with the relay. Except for one-way shared relaying with BS cooperation, we consider a single shared relay as depicted in our system models in conjunction with a single tier, that is, 3 cells, network. As in [7], we assume arbitrary scheduling and orthogonal signaling inside each sector (corresponding to a single subchannel of the OFDM waveform), and that the sum rate is calculated over three users for the various schemes. Table 1 shows the general parameters used throughout the simulations, many of which are unchanged from our previous work. Naturally, since we are concerned about interference management schemes, the network transmit powers will dictate interference powers throughout the network that prominently influence the performance. To quantify this interference and better interpret the simulations we give a brief overview of our channel models below, before presenting the results.

5.1. Channel Models. We adopt the channel models based on modifications of COST 231 (Walfisch-Ikegami) as proposed for the evaluation and comparison of relay-based IEEE 802.16j deployments. Note that the channel between each sector in each cell to the relay is a single-input multipleoutput channel (SIMO). Here, we model the link between the $j$ th sector of the $i$ th BS (cell) to the $N_{r}$-antenna shared relay terminal as $\mathbf{h}_{i j} \triangleq \sqrt{\alpha_{i j}} \widetilde{\mathbf{h}}_{i j}$, where $\widetilde{\mathbf{h}}_{i j} \sim \mathcal{C} \mathcal{N}\left(\mathbf{0}_{M}, \mathbf{I}_{M}\right)$ captures the small-scale fading, with the assumption of sufficient scattering in the cell, while $\alpha_{i j}$ captures the path loss (and possibly shadowing). $\alpha_{i j}$ is a function of the system parameters, such as carrier frequency (We assume a narrowband single carrier system.), and also of the relative distances between the terminals in the network. Similarly, the channel between the $j$ th MS in the $i$ th cell to the relay is $\mathbf{g}_{i j} \triangleq$ $\sqrt{\beta_{i j}} \tilde{\mathbf{g}}_{i j}$, where $\tilde{\mathbf{g}}_{i j} \sim \mathcal{C} \mathcal{N}\left(\mathbf{0}_{M}, \mathbf{I}_{M}\right)$, and $\beta_{i j}$ is the path loss. The IEEE $802.16 \mathrm{j}$-COST-231 model provides various categories of modeling (types $A$ through $J$ ) providing empirically derived equations for $\alpha_{i j}$ and $\beta_{i j}$ for various topological configuration such as line of sight (LOS) and nonline of sight (NLOS) channels, hilly, flat and heavy tree density terrains, above and below roof top terminal mountings (ART) and (BRT), urban and suburban city densities, and so forth. The choice of the category depends on the geographical characteristics of the specific region in which the system is to be deployed. The descriptions of each category may be found in the latest version of the "Multi-hop Relay System Evaluation Methodology".

Here, we choose an urban environment with fixed infrastructure at a carrier frequency of $2 \mathrm{GHz}$. The BSs and relay are located at above roof-top levels at a height of 30 and 15 meters, respectively, while each MS is located on street level, that is, below roof-top, at a height of 1 meter. The distance from each BS to the shared relay is $r_{i}=876$ meters (the cell radius) and the MSs are located at a distance of $0<d_{i j}<876$ meters from their respective sectors. The BS-RS links are categorized as type $H$ channels since they are ART-ART while the RS-MS links are categorized as type $E$ since the MSs are BRT. The path loss models also include power losses owing to antenna pattern gains, that is, directivity gains, where each BS is assumed to create a 6-beam patterns with $0 \mathrm{~dB}$ gain in the direction of the shared relay while we assume the relay and MSs use omnidirectional patterns. For example, the BS beam at an angle of $180^{\circ}$ from the shared relay provides a $23 \mathrm{~dB}$ power loss in the direction of the relay terminal. Note that such a large power-loss (also known as front-to-back ratio.) is welcoming here since (with universal frequency reuse) this sector is effectively creating interference into sector 1 , that is, the sector of interest. Table 2 summarizes the various parameters discussed above. The resulting path loss variables that account for all these parameters, are given in Table 2 where the sector of interest (least path loss) is highlighted. We point out that with the given transmit powers of Table 1, the cellular system is interference limited as apposed to noise limited. (This can be seen, for example, by calculating the average total interference from the BS to the relay as $\sigma_{\zeta_{b}}^{2}=(1 / M) \mathbb{E}\left\{\zeta_{b} \zeta_{b}^{*}\right\}=3 P_{b} \sum_{j=2}^{6} \alpha_{1 j}=-69.3 \gg N_{0} \mathrm{dBm}$. Similarly for the interference from the MSs to the relay we have $\sigma_{\zeta_{m}}^{2}=P_{m} \sum_{j=2}^{6} \beta_{1 j} / M=-98.4 \gg N_{0} \mathrm{dBm}$.)

5.2. Results. We now present the simulation results based on our channel models. Table 3 serves as a quick reference, summarizing the sum rate expressions and equations in the paper.

5.2.1. User Positioning. Given our path loss model, the position of the users is expected to influence the performence. To quantify this effect we simulate 2, 000 channel realizations and compute the average sum rate in the DL and UL within the sectors of interest pertaining to our schemes. For each channel (and noise) realization the MSs in the 
TABLE 2: Path loss coefficients

\begin{tabular}{|c|c|c|c|c|c|c|}
\hline$i=1,2,3$ & $j=1$ & $j=2$ & $j=3$ & $j=4$ & $j=5$ & $j=6$ \\
\hline$\alpha_{i j}(\mathrm{~dB})$ & -98.2 & -121.7 & -121.7 & -121.7 & -121.7 & -121.7 \\
\hline
\end{tabular}
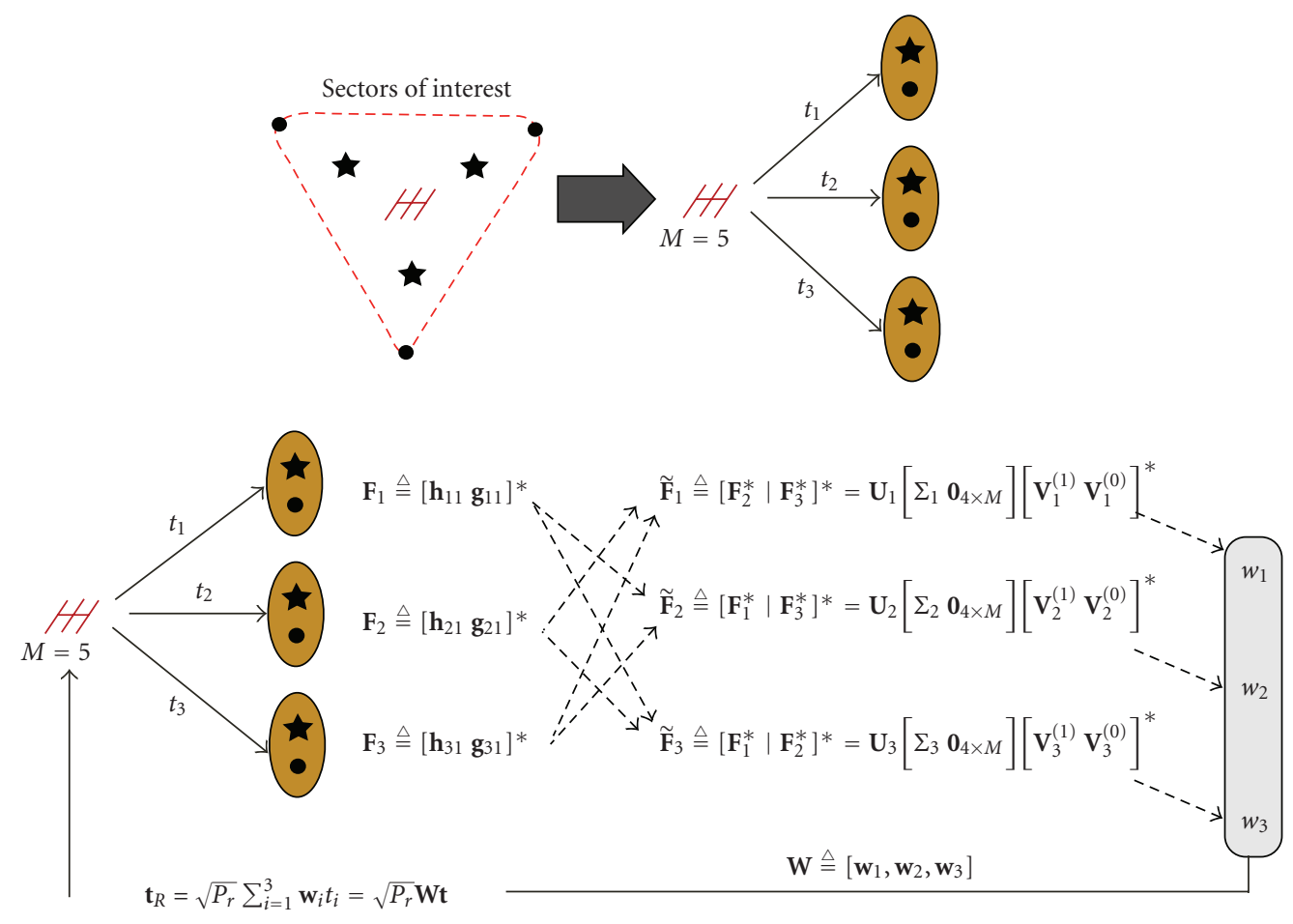

FIGURE 3: Operations of two-way block diagonalization at shared relay via SVD.

TABLE 3: Sum rate references for proposed schemes.

\begin{tabular}{lcc}
\hline Scheme & DL sum rate & UL sum rate \\
\hline one-way \\
\hline One-way shared & $R_{\text {shared }}^{\mathrm{DL}}(5)$ & $R_{\text {shared }}^{\mathrm{UL}}(6)$ \\
One-way shared w/ BS coop. & $R_{\text {coop }}^{\mathrm{DL}}(8)$ & $R_{\text {coop }}^{\mathrm{UL}}=R_{\text {shared }}^{\mathrm{UL}}$ \\
One-way 802.16j & $R_{\text {nonshared }}^{\mathrm{DL}}(17)$ & $R_{\text {nonshared }}^{\mathrm{UL}}(18)$ \\
\hline \multicolumn{3}{c}{ two-way } \\
\hline Two-way DSOF & $R_{\mathrm{DSOF}}^{\mathrm{DL}}(26)$ & $R_{\mathrm{DSOF}}^{\mathrm{UL}}(27)$ \\
Two-way ASF & $R_{\mathrm{ASF}}^{\mathrm{DL}}(35)$ & $R_{\mathrm{ASF}}^{\mathrm{UL}}(33)$ \\
Two-way ASOF & $R_{\mathrm{ASOF}}^{\mathrm{DL}}(39)$ & $R_{\mathrm{ASOF}}^{\mathrm{UL}}(38)$ \\
\hline
\end{tabular}

sectors of interest are positioned at a fixed distance from their respective base stations and are given a random phase location within that sector while all other MS's locations are chosen uniformly (in distance and phase) within their own sectors. Figures 4 and 5 show the sum rate performances versus the MS distance from the BS in the sectors of interest. Note that the right section of these plots correspond to the users being located at the cell edge. Several observations may be made here. The two-way DSOF is superior to all other schemes as it eliminates interference in both phases of transmission. The amplify and forward version of this scheme, that is, ASOF, is also effective at the cell edge where the average intersector interference is expected to be small. The nonshared relay performance peaks at an intermediate location which is expected given the relay positions and the shared relay surpasses this performance at the cell edge, both with and without BS coordination. Finally the two-way ASF is inferior as it lacks any interference management and simply forwards interference. As expected, the performance here is similar to the scheme in [7] where a naive AF protocol was considered. A similar trend holds for the performance in the UL in Figure 5.

Recall that the decode and forward protocols, such as the one-way shared relay protocol, amounted to the minimum rates achieved in two separate phases. For example, for the two-way DSOF from (26) we had $R_{\mathrm{DSOF}}^{\mathrm{DL}}=$ $(1 / 3) \min \left\{R_{1}^{\mathrm{DL}}, R_{3}^{\mathrm{DL}}\right\}$ and Figure 4 did not show the individual rates $R_{1}^{\mathrm{DL}}, R_{3}^{\mathrm{DL}}$ but instead ploted the resulting $R_{\mathrm{DSOF}}^{\mathrm{DL}}$. Figure 6 shows a break-down of performance via the two individual rates for the two-way DSOF scheme. As the MS moves away from the BS and toward the RS, that is, cell edge, $R_{3}^{\mathrm{DL}}$ increases due to less path loss in the MS-RS link. After a certain point, for example, $600 \mathrm{~m}$ in this figure, $R_{3}^{\mathrm{DL}}$ effectively overtakes $R_{1}^{\mathrm{DL}}$ and a bottleneck is created from the BS-RS link. In summary, this figure shows that the performance is limited by phase III when the MSs is away from the cell edge and by phase I when it is near the cell edge. Therefore one way to improve the performance further is to 


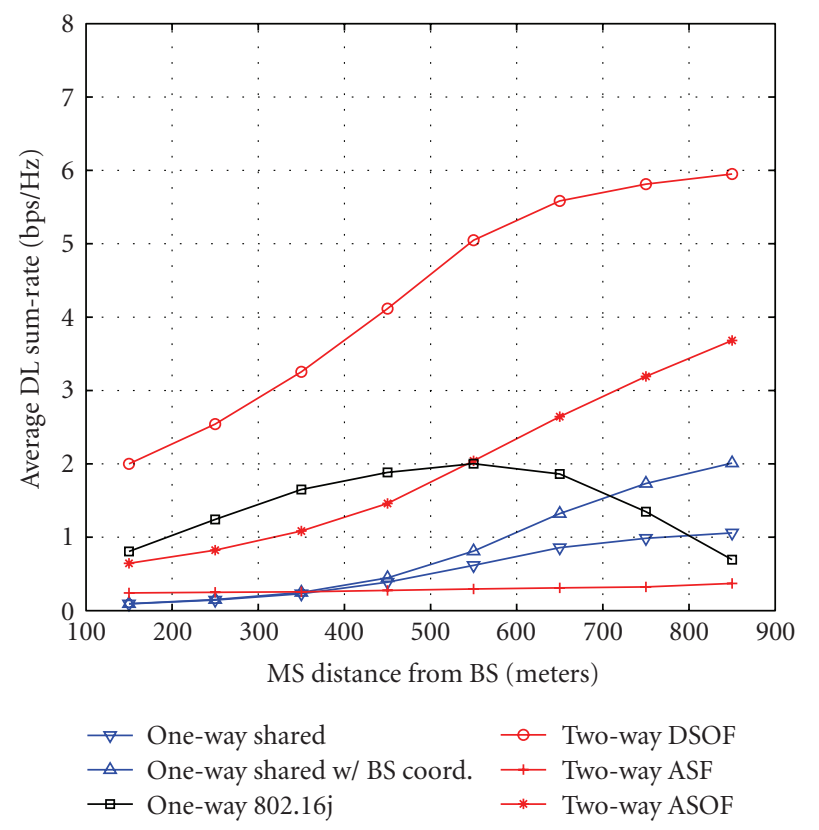

FIGURE 4: DL sum rate performances versus MS distance from BS.

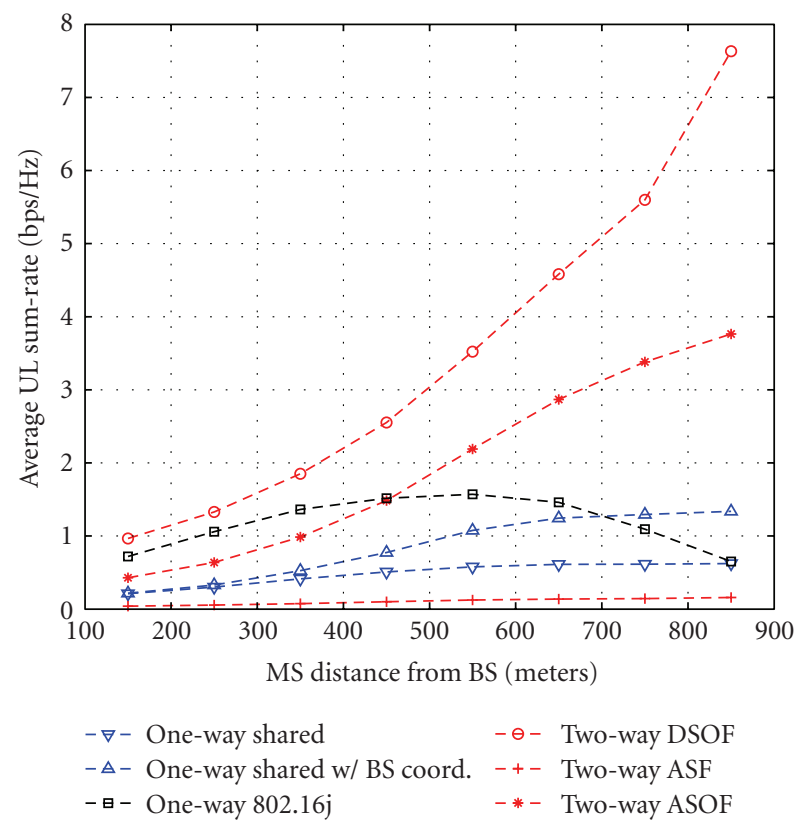

FigURE 5: UL sum rate performance versus MS distance from BS.

increase the relay transmit power when the MS is away from the cell edge and to increase the BS transmit power when the MS is near the cell edge.

5.2.2. Cell Edge Performance. The simulations above (particularly Figure 6) showed that the relay power can have significant effects on the end performance. While the relay power was fixed at $37 \mathrm{dBm}$ in those simulations we now look at the effects of varying relay power when the MSs are located at the cell edge. Figures 7 and 8 show the sum rate

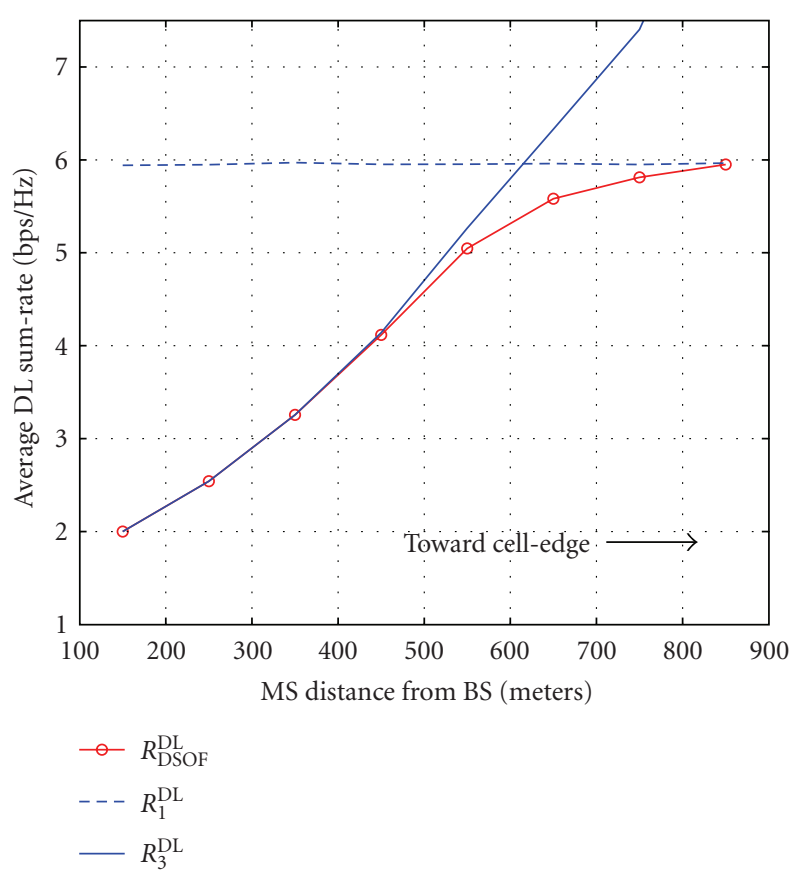

FIGURE 6: Performance break down of phases I and III for proposed two-way DSOF where $R_{\mathrm{DSOF}}^{\mathrm{DL}}=(1 / 3) \min \left\{R_{1}^{\mathrm{DL}}, R_{3}^{\mathrm{DL}}\right\}$.

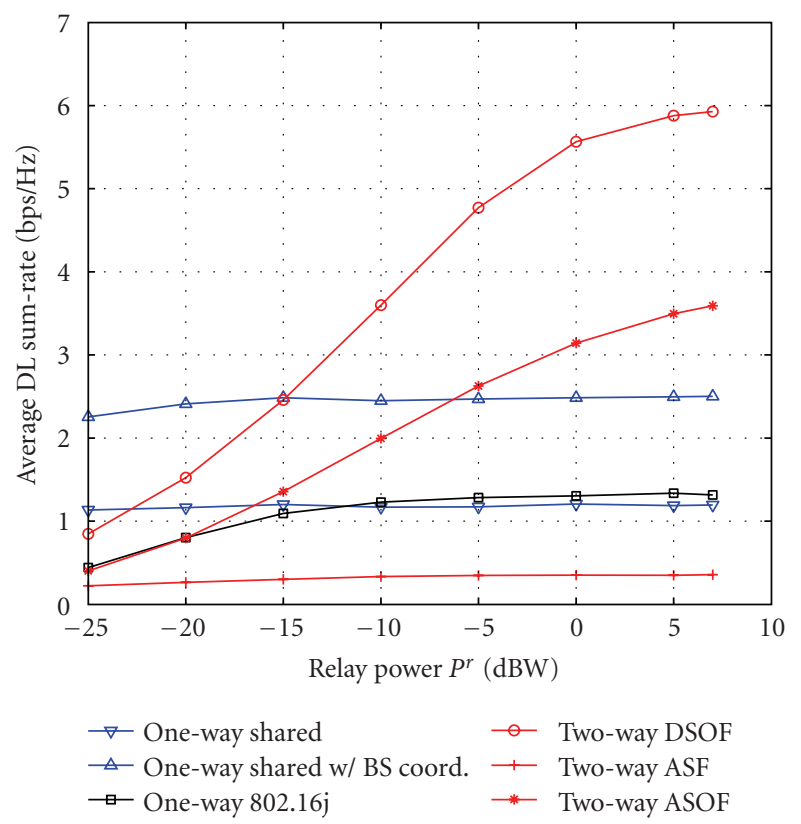

FIGURE 7: UL sum rate performance versus average relay transmit power.

performance as a function of relay transmit power for the proposed schemes. Increasing the relay transmit power is expected to improve performance especially when the relay is employing a strong interference cancelation scheme. The plots here show again how the two-way DSOF strategy is superior in this regard, specially at high $P^{r}$. Finally, we note that this performance gain comes at the expense of higher 


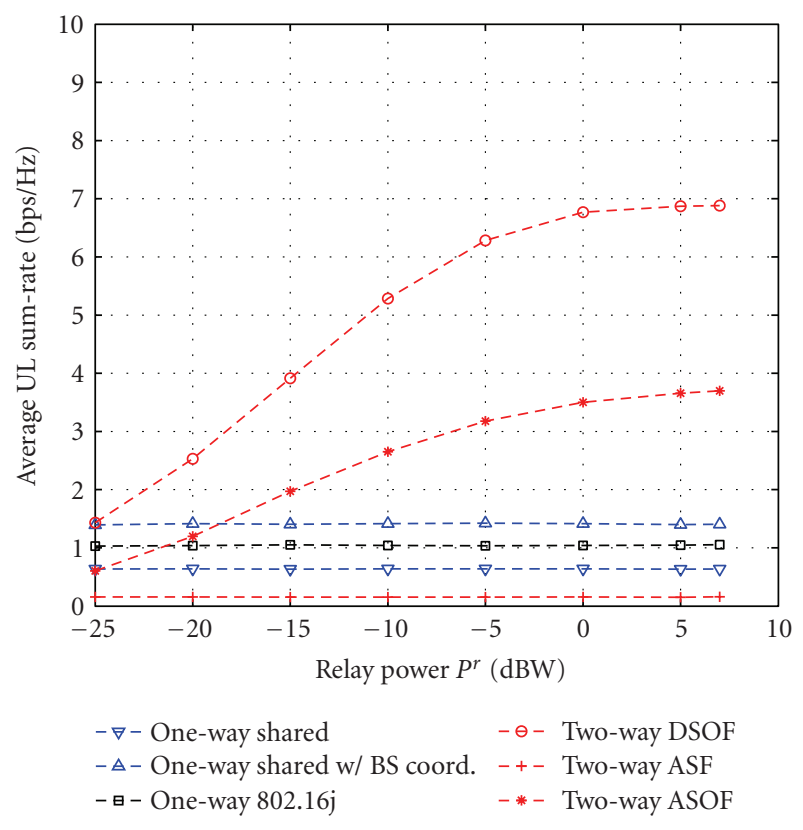

FIGURE 8: UL sum rate performance versus average relay transmit power.

transmit complexity, that is, block diagonalization, and the use of more antennas at the relay compared to the one-way counterparts.

\section{Conclusion}

Relay terminals are expected to play an important role in next generation cellular wireless deployments around the globe. Such relays are expected to improve link performance via sophisticated reception and/or transmission techniques as well as involvement in network interference management schemes. Previously we had introduced the shared relay concept, in which a single multi-antenna terminal is shared between several base stations. In this paper we extended that concept to incorporate several interference management schemes in the physical layer at the relays. We considered both one-way relaying and, the spectrally efficient twoway relaying categories. For each category we proposed several relay processing schemes to remove interference within the network. In some cases we also extended to include partial or full base station coordination, further improving the sum rate performances. We conducted linklevel simulations to assess and compare the performance of the schemes concluding that even simple relay processing such as zero forcing helps to significantly reduce interference and improve sum rate. Moreover, sophisticated techniques such as two-way relaying with block diagonalization surpass other techniques, specially when the relay is operating at high transmit power. The improvements here come at the cost of increased complexity not only at the relay but also at the receiving terminals by virtue of self-interference. The performance of the schemes were not optimized here. For example, given the high transmit asymmetry of the base stations and mobile stations in the network, power loading at the relay for dividing the power between transmission protocols is expected to improve performance. We believe this to be a promising venue for future work.

\section{Acknowledgment}

This work was supported by a gift from Huawei Technologies, Inc.

\section{References}

[1] S. W. Peters and R. W. Heath Jr., "The future of WiMAX: multihop relaying with IEEE $802.16 \mathrm{j}$," IEEE Communications Magazine, vol. 47, no. 1, pp. 104-111, 2009.

[2] S. Sesia, I. Toufik, and M. Baker, LTE, The UMTS Long Term Evolution: From Theory to Practice, John Wiley \& Sons, New York, NY, USA, 2009.

[3] D. Astély, E. Dahlman, A. Furuskär, Y. Jading, M. Lindström, and S. Parkvall, "LTE: The evolution of mobile broadband," IEEE Communications Magazine, vol. 47, no. 4, pp. 44-51, 2009.

[4] E. Dahlman, S. Parkvall, and J. Skold, 3G Evolution: HSPA and LTE for Mobile Broadband, Academic Press, Edinburg, UK, 2007.

[5] J. Lee, J.-K. Han, and J. Zhang, "MIMO technologies in 3GPP LTE and LTE-advanced," EURASIP Journal on Wireless Communications and Networking, vol. 2009, pp. 1-10, 2009.

[6] S. Parkvall, E. Dahlman, A. Furuskär et al., "LTE-Advanced Evolving LTE towards IMT-Advanced," in Proceedings of the 68th Semi-Annual IEEE Vehicular Technology Conference (VTC '08), pp. 1-5, September 2008.

[7] S. W. Peters, A. Y. Panah, K. T. Truong, and R. W. Heath Jr., "Relay architectures for 3GPP LTE-advanced," EURASIP Journal on Wireless Communications and Networking, vol. 2009, p. 1, 2009.

[8] G. Boudreau, J. Panicker, N. Guo, R. Chang, N. Wang, and S. Vrzic, "Interference coordination and cancellation for $4 \mathrm{G}$ networks," IEEE Communications Magazine, vol. 47, no. 4, pp. 74-81, 2009.

[9] M. Rahman, H. Yanikomeroglu, and W. Wong, "Interference avoidance with dynamic inter-cell coordination for downlink LTE system," in Proceedings of the IEEE Wireless Communications and Networking Conference (WCNC'09), April 2009.

[10] J. G. Andrews, W. Choi, and R. W. Heath Jr., "Overcoming interference in spatial multiplexing mimo cellular networks," IEEE Wireless Communications, vol. 14, no. 6, pp. 95-104, 2007.

[11] J. Ellenbeck, C. Hartmann, and L. Berlemann, "Decentralized inter-cell interference coordination by autonomous spectral reuse decisions," in Proceedings of the 14th European Wireless Conference (EW'08), pp. 1-7, June 2008.

[12] X. Qiu and K. Chawla, "Intra-cell interference management for fixed broadband wireless systems," in Proceedings of the 48th IEEE Vehicular Technology Conference (VTC'98), pp. 914919, May 1998.

[13] G. J. Foschini, K. Karakayali, and R. A. Valenzuela, "Coordinating multiple antenna cellular networks to achieve enormous spectral efficiency," IEE Proceedings: Communications, vol. 153 , no. 4, pp. 548-555, 2006. 
[14] S. Jing, D. N. C. Tse, J. B. Soriaga, J. Hou, J. E. Smee, and R. Padovani, "Multicell downlink capacity with coordinated processing," EURASIP Journal on Wireless Communications and Networking, vol. 2008, Article ID 586878, 2008.

[15] M. K. Karakayali, G. J. Foschini, and R. A. Valenzuela, "Network coordination for spectrally efficient communications in cellular systems," IEEE Wireless Communications, vol. 13, no. 4, Article ID 1678166, pp. 56-61, 2006.

[16] H. Zhang and H. Dai, "Cochannel interference mitigation and cooperative processing in downlink multicell multiuser MIMO networks," EURASIP Journal on Wireless Communications and Networking, vol. 2004, no. 2, pp. 222-235, 2004.

[17] J. Ponniah and L.-L. Xie, "An achievable rate region for the two-way two-relay channel," in Proceedings of the IEEE International Symposium on Information Theory (ISIT '08), pp. 489-493, July 2008.

[18] M. Chen and A. Yener, "Power allocation for F/TDMA multiuser two-way relay networks," IEEE Transactions on Wireless Communications, vol. 9, no. 2, pp. 546-551, 2010.

[19] C. Eşli and A. Wittneben, "One-and two-way decode-andforward relaying for wireless multiuser MIMO networks," in Proceedings of the IEEE Global Telecommunications Conference (GLOBECOM '08), pp. 1-6, December 2008.

[20] V. Havary-Nassab, S. Shahbazpanahi, and A. Grami, "Optimal network beamforming for bi-directional relay networks," IEEE Transactions on Signal Processing, vol. 58, no. 3, pp. 1238-1250, 2010.

[21] M. Chen and A. Yener, "Interference management for multiuser two-way relaying," in Proceedings of the 42nd Annual Conference on Information Sciences and Systems (CISS '08), pp. 246-251, March 2008.

[22] B. Rankov and A. Wittneben, "Achievable rate regions for the two-way relay channel," in Proceedings of the IEEE International Symposium on Information Theory (ISIT '06), pp. 1668-1672, July 2006. 